\title{
A VERY LARGE ARRAY 3.6 CENTIMETER CONTINUUM SURVEY OF GALACTIC WOLF-RAYET STARS
}

\author{
C. $\mathrm{CAPPA}^{1}$ \\ Instituto Argentino de Radioastronomía, C.C. 5, 1894 Villa Elisa, Argentina; and Facultad de Ciencias Astronómicas y Geofísicas, \\ Universidad Nacional de La Plata, Paseo del Bosque s/n, 1900 La Plata, Argentina; ccappa@fcaglp.fcaglp.unlp.edu.ar \\ W. M. Goss \\ National Radio Astronomy Observatory, P.O. Box O, Socorro, NM 87801; mgoss@aoc.nrao.edu \\ AND \\ K. A. van der Hucht \\ SRON National Institute for Space Research, Sorbonnelaan 2, NL-3584 CA Utrecht, Netherlands; k.a.van.der.hucht@sron.nl \\ Received 2003 May 28; accepted 2004 January 23
}

\begin{abstract}
We report the results of a survey of radio continuum emission of Galactic Wolf-Rayet (WR) stars north of $\delta=-46^{\circ}$. The observations were obtained at $8.46 \mathrm{GHz}(3.6 \mathrm{~cm})$ using the Very Large Array, with an angular resolution of $\sim 6^{\prime \prime} \times 9^{\prime \prime}$ and typical rms noise of $\sim 0.04 \mathrm{mJy}^{\text {beam }}{ }^{-1}$. Our survey of 34 WR stars resulted in 15 definite and five probable detections, 13 of these for the first time at radio wavelengths. All detections are unresolved $\left(\theta \lesssim 5^{\prime \prime}\right)$. Time variations in flux are confirmed in the cases of WR 98a, 104, 105, and 125. WR 79a and WR 89 are also variable in flux, and we suspect they are also nonthermal emitters. Thus, of our sample $20 \%-$ $30 \%$ of the detected stars are nonthermal emitters. Average mass-loss rate determinations obtained excluding definite and suspected nonthermal cases give similar values for WN (all subtypes) and WC5-7 stars $\left[\dot{M}(\mathrm{WN})=(4 \pm 3) \times 10^{-5} M_{\odot} \mathrm{yr}^{-1}\right.$ and $\left.\dot{M}(\mathrm{WC} 5-7)=(4 \pm 2) \times 10^{-5} M_{\odot} \mathrm{yr}^{-1}\right]$, while a lower value was obtained for WC8-9 stars $\left[\dot{M}(\mathrm{WC} 8-9)=(2 \pm 1) \times 10^{-5} M_{\odot} \mathrm{yr}^{-1}\right]$. Uncertainties in stellar distances largely contribute to the observed scatter in mass-loss rates. Upper limits to the mass-loss rates were obtained in cases of undetected sources and for sources that probably show additional nonthermal emission.
\end{abstract}

Key words: radio continuum: stars — stars: mass loss — stars: Wolf-Rayet

\section{INTRODUCTION}

Wolf-Rayet (WR) stars are the evolved descendants of massive O-type progenitors $\left(M_{i}>22 M_{\odot}\right)$ and are considered to be the immediate precursors of supernovae of Types $\mathrm{Ib}$ and Ic (e.g., Maeder 1981; Meynet \& Maeder 2003). They are characterized by powerful stellar winds with terminal velocities $v_{\infty} \simeq 400-5500 \mathrm{~km} \mathrm{~s}^{-1}$ (e.g., Prinja et al. 1990; Eenens \& Williams 1994; Kingsburgh et al. 1995; for an overview see van der Hucht 2001, Table 15, col. [14]), and mass-loss rates in the range $(1-5) \times 10^{-5} M_{\odot} \mathrm{yr}^{-1}$ (e.g., Abbott et al. 1986, hereafter AB86; Leitherer et al. 1995, 1997, hereafter LC95, LC97; Chapman et al. 1999, hereafter CL99). Because of these properties WR stars are not only interesting in themselves, but also important species for studies of chemical enrichment and Galactic evolution (e.g., Dray et al. 2003; De Donder \& Vanbeveren 2003). For general reviews of the WR phenomenon, see, e.g., van der Hucht et al. (1992) and Maeder \& Conti (1994).

The strong stellar winds of WR stars reveal themselves in the electromagnetic spectrum from the UV (P Cygni profiles of resonance lines) to the IR/radio range (free-free radiation). Therefore, an important diagnostic tool to obtain information about stellar wind properties of massive stars is the study of the radio continuum emission from their close environs. Radio observations provide a reliable method for determining stellar mass-loss rates if the emission is thermal

\footnotetext{
${ }^{1}$ Member of Carrera del Investigador Científico, CONICET, Argentina.
}

in origin, since the measured radio flux density is related to the mass-loss rate by an analytical expression (e.g., Wright \& Barlow 1975; Panagia \& Felli 1975). This emission is the free-free radiation originating in the ionized wind, expanding at constant velocity. It arises from the outer parts of the wind envelope and is partially optically thick in the radio region, showing positive spectral indices $(\alpha \geq+0.6$, where $\left.S_{\nu} \propto \nu^{\alpha}\right)$.

However, the last decade has seen an increasing number of discoveries of WR stars with nonthermal radio emission in addition to their free-free emission. Nonthermal emission is thought to be produced by electrons accelerated in strong shocks in the winds of single stars (White 1985) or in the shocked wind-wind collision region between massive $\mathrm{WR}+\mathrm{O}$ binaries (e.g., Williams et al. 1990; Eichler \& Usov 1993). Unlike thermal emission, nonthermal emission has a negative spectral index and can be variable. Van der Hucht et al. (1992) suggested that all cases of WR nonthermal radio emission correspond to $\mathrm{WR}+\mathrm{OB}$ colliding-wind binaries, which has been corroborated by Dougherty \& Williams (2000).

Because of their relatively large distances (van der Hucht 2001 ), the number of WR radio continuum detections was limited to only about 44 cases (AB86, LC95, LC97, and CL99). The Very Large Array (VLA) survey by AB86, which also quotes previous observations, contains radio data for $40 \mathrm{WR}$ stars at $\delta>-47^{\circ}$ with 27 detections using the VLA, with rms noise values of $0.06-0.23 \mathrm{mJy} \mathrm{beam}^{-1}$. They found $77 \%$ probable or definite thermal wind sources and $12 \%$ probable or definite nonthermal sources. In the southern hemisphere 
TABLE 1

Selected Wolf-Rayet Stars

\begin{tabular}{|c|c|c|c|c|c|c|}
\hline $\begin{array}{l}\text { WR No. } \\
\text { (1) }\end{array}$ & $\begin{array}{l}\text { Other Designation } \\
\text { (s) } \\
\text { (2) }\end{array}$ & $\begin{array}{c}\text { Spectral Type } \\
\text { (3) }\end{array}$ & $\begin{array}{c}d \\
(\mathrm{kpc}) \\
(4)\end{array}$ & $\begin{array}{c}\text { Binary Status } \\
\text { (5) }\end{array}$ & $\begin{array}{c}P \\
\text { (days) } \\
(6)\end{array}$ & $\begin{array}{c}v_{\infty} \\
\left(\mathrm{km} \mathrm{s}^{-1}\right) \\
(7)\end{array}$ \\
\hline $4 \ldots \ldots$ & HD 16523, V493 Per & WC5+? & 2.44 & SB1, no d.e.1. & 2.4 & 1900 \\
\hline 5 & HD 17638 & WC6 & 1.91 & $\ldots$ & . & 2100 \\
\hline $8 \ldots \ldots \ldots \ldots \ldots \ldots \ldots$ & HD 62910 & WN7/WCE+? & 3.47 & SB1 & 38.4 & 1590 \\
\hline $12 \ldots \ldots \ldots \ldots \ldots \ldots \ldots \ldots \ldots \ldots \ldots \ldots$ & Ve5-5 & $\mathrm{WN} 8 \mathrm{~h}+?$ & 5.04 & SB1, no d.e.l. & 23.9 & 1100 \\
\hline $79 \mathrm{a}$ & HD 152408 & WN9ha & 1.99 & VB $\left(6^{\prime \prime} .9\right)$ & $\ldots$ & 935 \\
\hline $79 \mathrm{~b}$ & HD 152386 & WN9ha & 2.90 & VB $\left(0^{\prime \prime} 55\right)$ & $\ldots$ & 1650 \\
\hline $80 \ldots \ldots \ldots \ldots$ & Wra 1581 & WC9d & 1.50 & . & $\ldots$ & 1200 \\
\hline$\ldots \ldots \ldots \ldots \ldots$ & He3-1316 & WC9 & 1.57 & . & . & 910 \\
\hline $82 \ldots \ldots \ldots \ldots \ldots$ & LS 11 & WN7(h) & 5.25 & $\ldots$ & 2.0 & 1100 \\
\hline $87 \ldots \ldots$ & LSS 4064 & $\mathrm{WN} 7(\mathrm{~h})+\mathrm{OB}$ & 2.88 & a, d.e.l. & $\ldots$ & 1400 \\
\hline 88 & Thé 1 & WC9 & 2.33 & $\ldots$ & $\ldots$ & 1125 \\
\hline 89 & AS 223 & $\mathrm{WN} 8 \mathrm{~h}+\mathrm{OB}$ & 2.88 & a, d.e.l., VB $(9.88)$ & $\ldots$ & 1600 \\
\hline $92 \ldots \ldots \ldots$ & HD 157451 & WC9 & 3.84 & $\ldots$ & $\ldots$ & 1100 \\
\hline $95 \ldots \ldots \ldots$ & He3-1434 & WC9d & 2.09 & $\ldots$ & $\ldots$ & 1100 \\
\hline 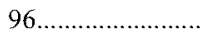 & LSS 4265 & WC9d & 3.58 & $\ldots$ & $\ldots$ & 1100 \\
\hline $98 \mathrm{a} \ldots \ldots \ldots \ldots \ldots \ldots$ & IRAS $17380-3031$ & WC8-9vd+? & 1.9 & CWB & 565 & $900^{1}$ \\
\hline $100 \ldots \ldots \ldots \ldots \ldots \ldots$ & HDE 318139 & WN7 & 4.61 & $\ldots$ & $\ldots$ & 1600 \\
\hline $103 \ldots \ldots \ldots \ldots \ldots$ & HD 164270, V4072 Sgr & WC $9 \mathrm{~d}+?$ & 2.21 & SB1, no d.e.1. & 1.8 & 1100 \\
\hline $104 \ldots \ldots \ldots \ldots \ldots$ & Ve2-45 & $\mathrm{WC} 9 \mathrm{~d}+\mathrm{B} 0.5 \mathrm{~V}$ & 2.3 & SB2, VB $(0.975)$ & 243.5 & 1220 \\
\hline $105 \ldots \ldots \ldots \ldots \ldots$ & Ve2-47 & WN9h & 1.58 & $\ldots$ & $\ldots$ & 700 \\
\hline $106 \ldots \ldots \ldots \ldots \ldots$ & HDE 313643 & WC9d & 2.32 & $\ldots$ & $\ldots$ & 1100 \\
\hline $113 \ldots \ldots \ldots \ldots \ldots$ & HD 168206, CV Ser & $\mathrm{WC} 8 \mathrm{~d}+\mathrm{O} 8-9 \mathrm{IV}$ & 1.79 & SB2 & 29.7 & 1700 \\
\hline $114 \ldots \ldots \ldots \ldots \ldots \ldots$ & HD 169010 & WC5+OB? & 2.00 & d.e.l. & $\ldots$ & 2000 \\
\hline $119 \ldots \ldots \ldots \ldots \ldots \ldots$ & Thé 2 & WC9d & 3.31 & $\ldots$ & $\ldots$ & 1200 \\
\hline $120 \ldots \ldots \ldots \ldots \ldots \ldots$ & Vyl-3 & WN7 & 3.56 & $\ldots$ & $\ldots$ & 1225 \\
\hline $121 \ldots \ldots \ldots \ldots \ldots \ldots . .$. & AS 320 & WC9d & 1.83 & $\ldots$ & $\ldots$ & 1100 \\
\hline $124 \ldots \ldots \ldots \ldots \ldots \ldots$ & 209 BAC, QR Sge & WN8h & 3.36 & SB1?, no d.e.l. & 2.7 & 710 \\
\hline $125 \ldots \ldots \ldots \ldots \ldots \ldots$ & IC14-36, V378 Vul & WC7ed+O9 III & 3.06 & $\mathrm{SB} 2$ & $>6600$ & 2900 \\
\hline $133 \ldots \ldots \ldots \ldots \ldots \ldots \ldots$ & HD 190918, V1676 Cyg & WN5+O9 I & 2.14 & SB2, VB $\left(5^{\prime \prime} 4\right)$ & 112.4 & 1800 \\
\hline $142 \ldots \ldots \ldots \ldots \ldots \ldots$ & Sand 5 & WO2 & 0.95 & (3) & $\ldots$ & $5500^{2}$ \\
\hline $143 \ldots \ldots \ldots \ldots \ldots \ldots$ & HD 195177 & $\mathrm{WC} 4+\mathrm{OB} ?$ & 1.07 & d.e.l. & . & 2750 \\
\hline $153 \mathrm{ab}$ & HD 211853, GP Cep & WN6/WCE +O6 I & 2.75 & & 6.7 & 1785 \\
\hline $155 \ldots \ldots \ldots \ldots \ldots$ & HD 214419, CQ Cep & $\mathrm{WN6}+\mathrm{O} 9 \mathrm{II}-\mathrm{Ib}$ & 2.75 & SB2 & 1.6 & 1400 \\
\hline $156 \ldots \ldots \ldots \ldots \ldots$ & $\mathrm{AC}+6038562$ & $\mathrm{WN} 8 \mathrm{~h}+\mathrm{OB} ?$ & 3.56 & d.e.l. & 6.5 & 660 \\
\hline
\end{tabular}

Notes.-Cols. (1) and (2): WR catalog number and any other designation(s); col. (3): spectral classification; col. (4): heliocentric distance; col. (5): the binary status, in case of visual binaries (VBs), the angular separation is indicated; col. (6): period; col. (7): terminal wind velocity. a: absorption lines present in optical spectrum; (d.e.l.): diluted emission lines.

All parameters and values from van der Hucht et al. 2001 except: (1) from Monnier et al. 2002; (2) from Kingsburgh et al. 1995.

LC95, LC97, and CL99 surveyed 36 WR stars $\left(\delta<-45^{\circ}\right)$ at two frequencies using the ATCA, with rms noise values of $0.10-0.15 \mathrm{mJy}$ beam $^{-1}$. Other WR radio continuum studies have been performed for WR 140 by Williams et al. (1987, 1990, 1994) and White \& Becker (1995); for WR 146 by Dougherty et al. $(1996,2000)$ and Setia Gunawan et al. (2000); for WR 147 by Moran et al. (1989), Churchwell et al. (1992), Davis et al. (1996), Williams et al. (1997), Skinner et al. (1999), Setia Gunawan et al. (2001), Watson et al. (2002), and Dougherty et al. (2003); for Galactic center WR stars by Lang et al. (2001) and Lang (2003); for WR 98a, 104, and 112 by Monnier et al. (2002); and for massive stars in Cyg $\mathrm{OB} 2$ by Setia Gunawan et al. (2003b). In these studies, some 70 out of the $\sim 250$ known Galactic WR stars (van der Hucht et al. 2001, 2003) have been investigated for radio continuum emission, resulting into 44 positive detections. The ATCA survey also indicates that at least $40 \%$ of the WR stars with measured radio spectral indices contain a nonthermal component at centimeter wavelengths, a percentage that, coincidentally, is equal to the percentage of $\mathrm{WR}+\mathrm{OB}$ binaries among the known Galactic WR stars (van der Hucht et al. 2001).
In this paper we report on the results of a survey of radio continuum emission from Galactic WR stars at $3.6 \mathrm{~cm}$ with higher sensitivity than available before. Our aim is to increase the sample of WR stars with radio continuum observations in order to derive mass-loss rates or upper limits and to identify nonthermal cases. As a first step, we performed observations at one frequency only. Consequently, spectral indices of detected sources can only be determined here using radio data at other wavelengths from the literature and may be influenced by variability. Thus, new radio continuum observations are important to find new thermal and nonthermal WR radio sources, the thermal ones providing mass-loss rates, crucial for testing existing wind models, and the nonthermal ones pointing to colliding winds in $\mathrm{WR}+\mathrm{OB}$ binaries.

\section{SELECTED WOLF-RAYET STARS}

To achieve these goals, we selected 33 WR stars from the catalog by van der Hucht (2001). To select the targets, we took into account all WR stars north of $\delta=-46^{\circ}$ and selected the ones with expected free-free flux densities $S_{\text {exp }}>0.075 \mathrm{mJy}$. $S_{\exp }$ was determined with the classical expressions by Panagia 


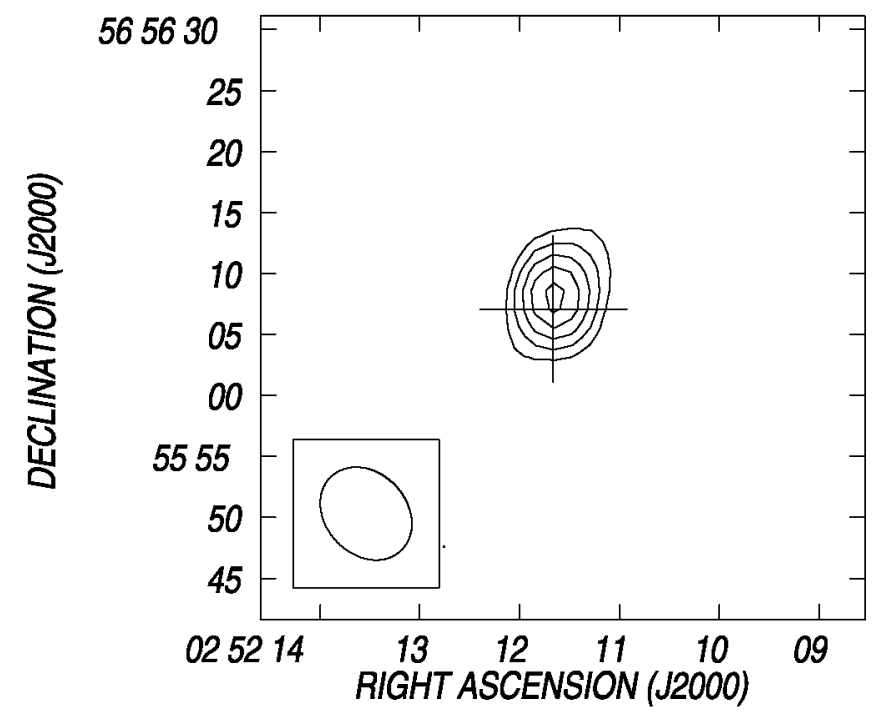

FIG. 1. Contour image for WR 5. The cross marks the optical position of the WR star, and the ellipse in one of the corners the synthesized beam. We note that the extent of the cross does not represent the uncertainty in the stellar position. Contour lines are -3 (dashed contour), 3, 4, 5, 6, and $7 \sigma(1 \sigma=0.025 \mathrm{mJy}$ beam ${ }^{-1}$ ).

\& Felli (1975) and Wright \& Barlow (1975) for the radio emission from an optically thick stellar wind flowing at constant velocity. To estimate these values, we adopt for all sources the same mass-loss rate, $\dot{M}=(1.5-2.0) \times 10^{-5} M_{\odot}$ $\mathrm{yr}^{-1}$, and stellar distances and terminal velocities from van der Hucht (2001).

With these assumptions, we found $S_{\text {exp }}>0.075 \mathrm{mJy}$ for 64 WR stars. Only 27 of these stars had previous radio detections, and radio flux density upper limits were available for 12 stars. We were then left with 37 stars that either had not been observed yet or had not been detected. We selected 26 of these stars as our targets, as listed in Table 1. In our sample we included some WR stars previously detected: WR 79a (HD 152804, detected with the VLA by Bieging et al. 1989, hereafter BA89), WR 81, 89, 98a, 104, 105, and 125. The detection of WR 81 by AB86 was doubtful. WR $98 \mathrm{a}, 104$,

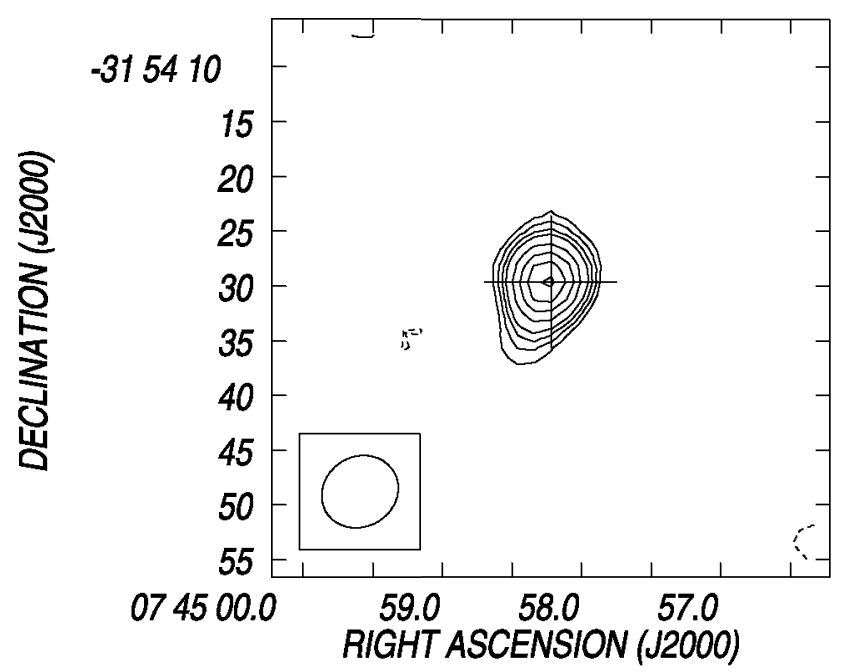

FIG. 2.-Contour image for WR 8 . Contour lines are -3 (dashed contour), $3,4,5,6,8,10,12$, and $14 \sigma\left(1 \sigma=0.030 \mathrm{mJy}^{\text {beam }}{ }^{-1}\right)$.

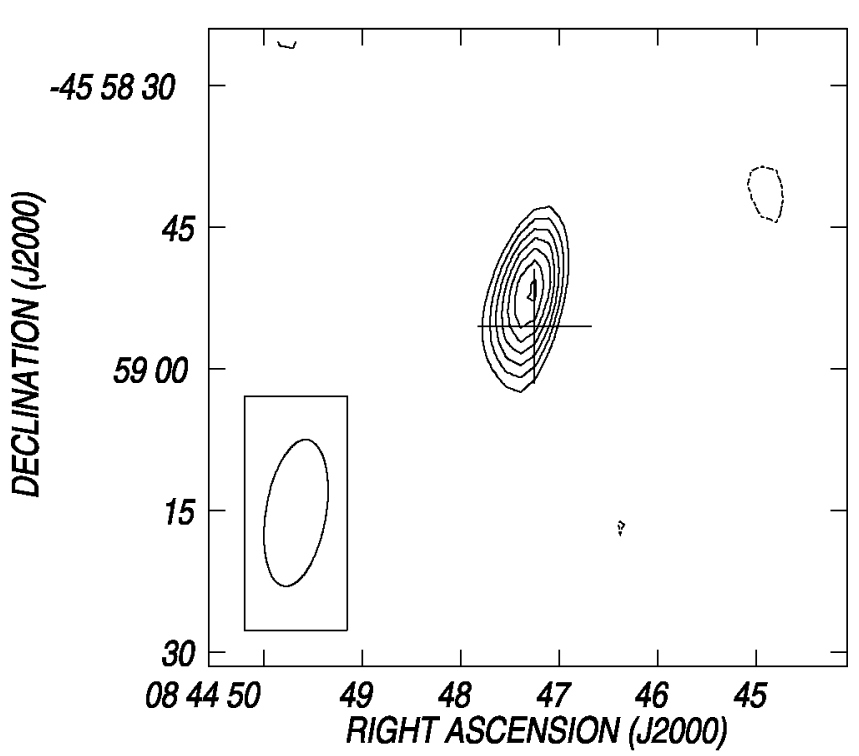

FIG. 3.-Contour image for WR 12. Contour lines are -3 (dashed contour), $3,4,5,6,7,8$, and $9 \sigma\left(1 \sigma=0.055 \mathrm{mJy}_{\text {beam }}^{-1}\right)$.

105 , and 125 are known nonthermal emitters and thus may vary in flux density.

To find new nonthermal candidates, we included binaries and suspected binaries listed by van der Hucht (2001). About $70 \%$ of the stars are closer than $3.0 \mathrm{kpc}$, while no stars beyond $5.3 \mathrm{kpc}$ are included.

\section{VLA OBSERVATIONS}

We performed radio continuum observations at $8.46 \mathrm{GHz}$ with the VLA in the direction of the selected WR stars. Thirtythree fields were observed on 2001 September 15 (during the move from the $\mathrm{C}$ to the DnC configuration), 2001 October 5 and 8 (in the DnC configuration) and 2001 November 12 (in the D configuration). With the exception of WR 89 , the field centers coincide with the stellar positions. The bandwidth was $50 \mathrm{MHz}$. The on-source observing time was about 25 minutes for each field to achieve a rms noise of $\sim 0.04 \mathrm{mJy} \mathrm{beam}^{-1}$. The synthesized beam is about $6^{\prime \prime} 1 \times 9^{\prime \prime} 0$ in all cases.

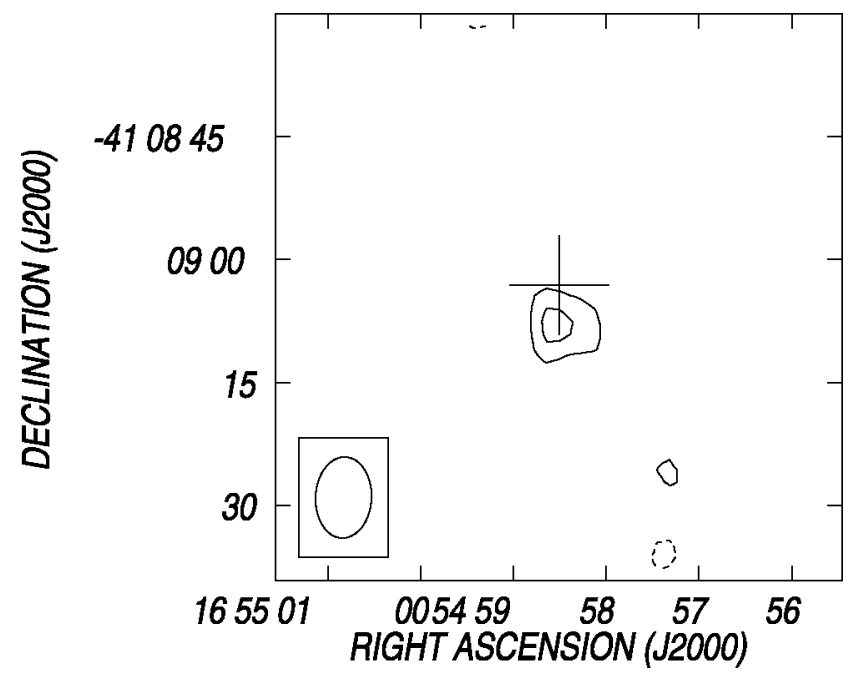

Fig. 4.-Contour image for WR 79a. Contour lines are -3 (dashed contour $), 3$, and $4 \sigma\left(1 \sigma=0.125 \mathrm{mJy}\right.$ beam $\left.^{-1}\right)$. 


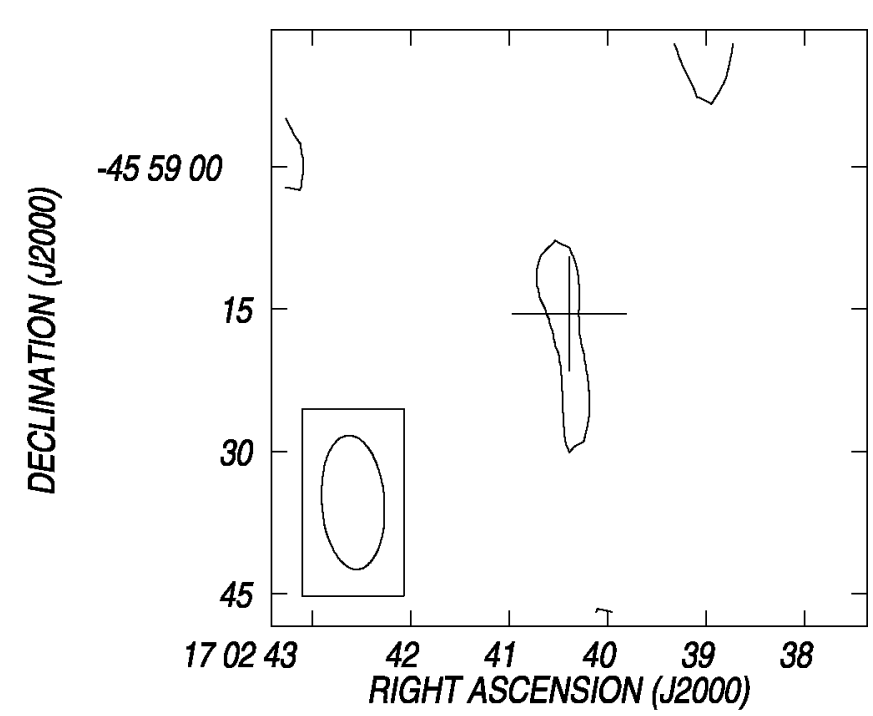

FIg. 5.-Contour image for WR 81. Contour lines are -3 (dashed contour) and $3 \sigma\left(1 \sigma=0.065 \mathrm{mJy}^{\text {beam }}{ }^{-1}\right)$.

The data were edited, calibrated, and imaged following a standard method using AIPS tasks. After editing the data were calibrated both in amplitude and phase. The primary flux density calibrators were $3 \mathrm{C} 48$ and $3 \mathrm{C} 286$. We used phase referencing, taking into account a selection of nearby point sources with precision positions $(0$ " 1 or better). The secondary calibrators were $0244+624,0747-331,0828-$ $375,1001-446,1604-446,1744-312,1751-253,1820-$ $254,1832-105,1851+005,1924+156,2015+371,2230+$ 697 , and $2250+558$. The fields corresponding to WR 4, 96, and 142 were also self-calibrated in phase, since peak flux densities within the primary beam exceed $10 \mathrm{mJy}^{\text {beam }}{ }^{-1}$. Primary beam correction was applied to the images corresponding to WR 89 and WR 105 . WR 89 is in the same field as WR $87,1.9$ far from the field center. The primary beam correction factor for WR 89 is 1.5 . In the case of WR 105 two additional pointlike sources, with no relation to the WR star, are detected close to the center of the field. Diffuse emission from extended sources detected in the fields of WR $87,104,106,113,114,124,125,142,143$, and $153 \mathrm{ab}$ was

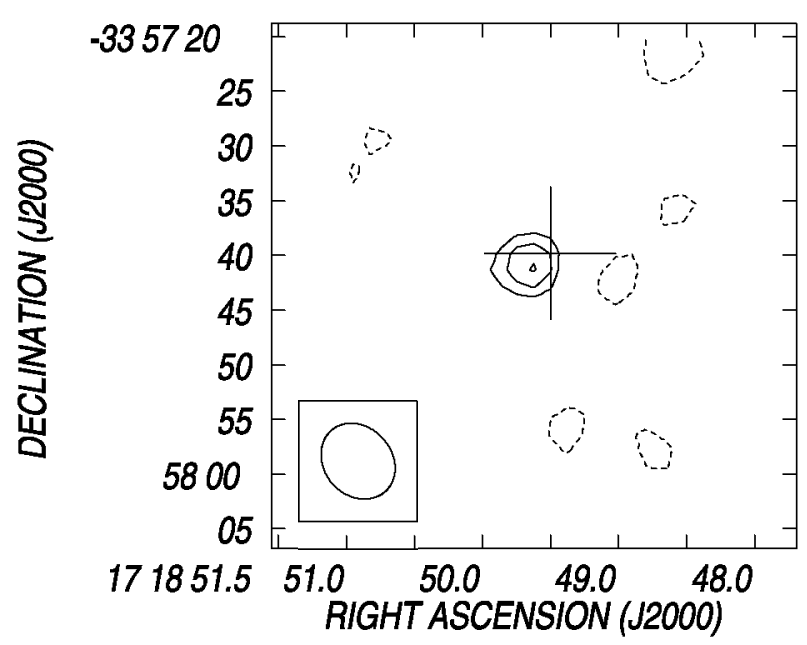

Fig. 6.-Contour image for WR 88. Contour lines are -3 (dashed contour), 3,4 , and $5 \sigma\left(1 \sigma=0.050 \mathrm{mJy}\right.$ beam $\left.^{-1}\right)$.

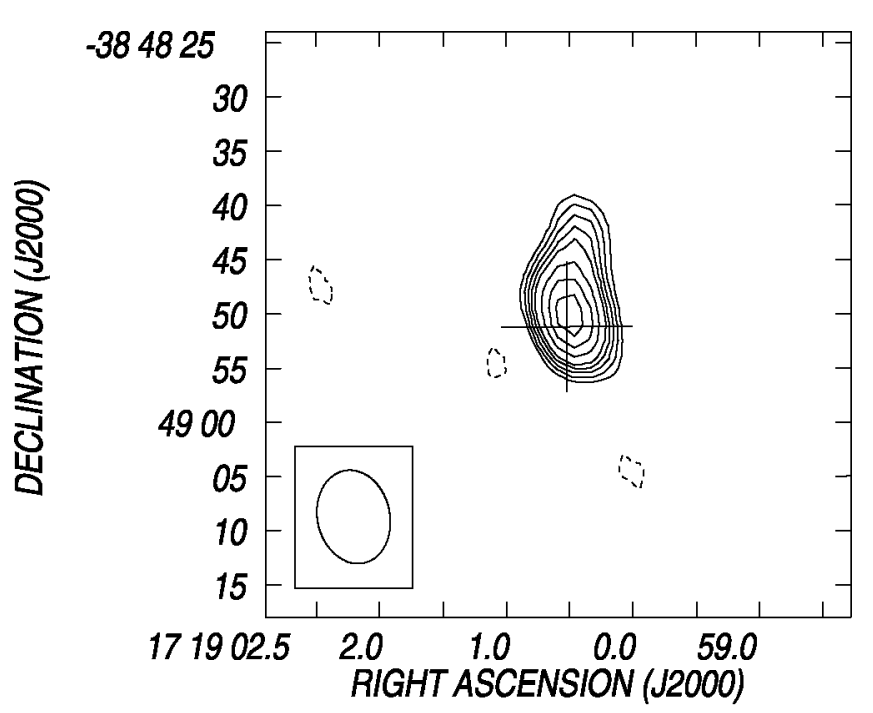

FIG. 7.-Contour image for WR 89. Contour lines are -3 (dashed contour), $3,4,5,6,7,9,11$, and $13 \sigma\left(1 \sigma=0.130 \mathrm{mJy} \mathrm{beam}^{-1}\right)$.

removed by applying a UV-RANGE $\geq 5 \mathrm{~K} \lambda$ (rejecting spatial scales $\simeq 40^{\prime \prime}$ ).

\section{OBSERVED RADIO EMISSION}

We report 15 definite and five probable detections at $3.6 \mathrm{~cm}$ out of the 34 observed WR stars. For 10 out of the 15 definite detections and three out of the five probable detections, this represents a first radio detection, while four definite detections had been previously identified at $3.6 \mathrm{~cm}$, and one had been detected previously at a different frequency. All 20 detections are unresolved. The images of the certain and probable detections are displayed in Figures 1-20. The synthesized beam of each field is shown in a corner of the contour plots.

Radio detections with signal-to-noise ratio $(\mathrm{S} / \mathrm{N}) \geq 5$, as derived from the Gaussian fitting, and close agreement between radio and optical positions were classified as definite detections, while probable detections correspond to sources with $\mathrm{S} / \mathrm{N} \approx 4$ (WR $81,95,114$, and 155 ) or with relatively large difference between the optical and the radio positions

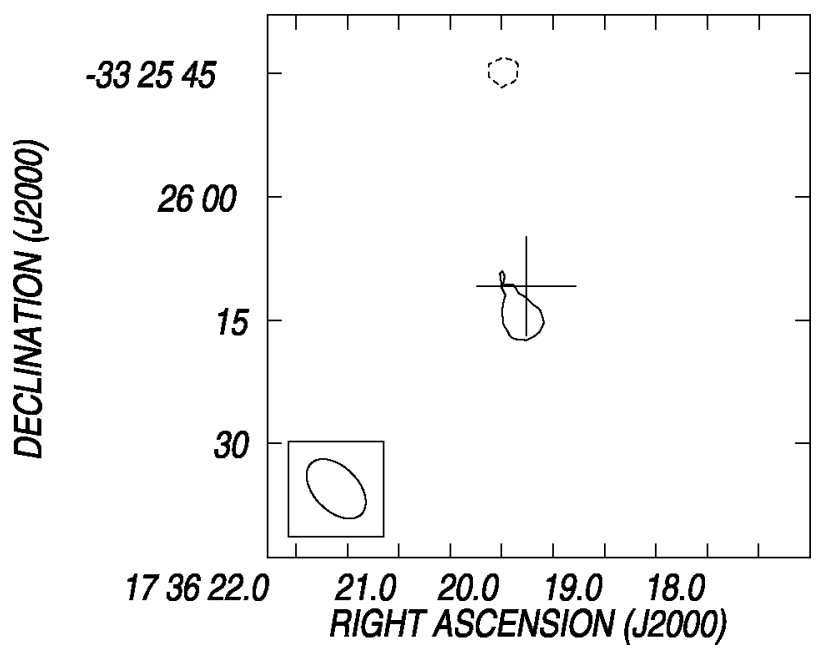

FIG. 8.-Contour image for WR 95. Contour lines are -3 (dashed contour) and $3 \sigma\left(1 \sigma=0.040 \mathrm{m.Jy}\right.$ beam $\left.^{-1}\right)$. 


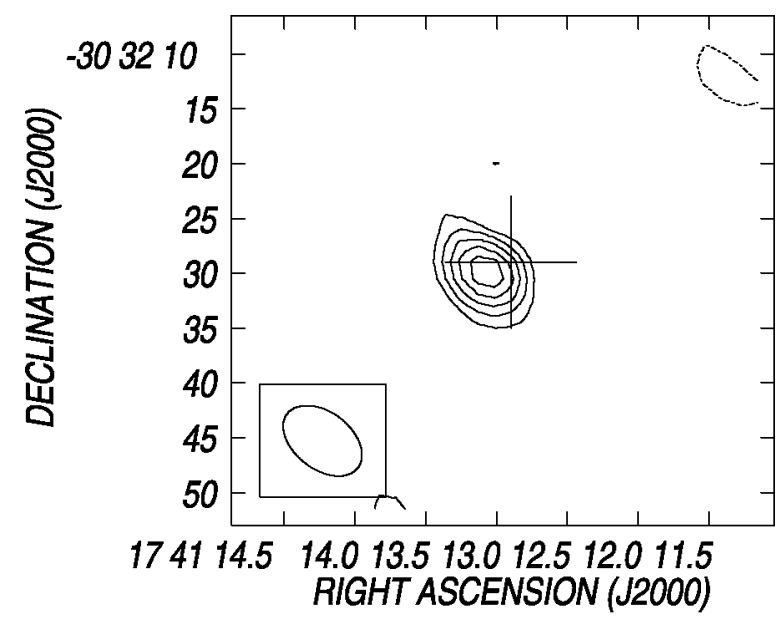

FIG. 9.-Contour image for WR 98a. Contour lines are -3 (dashed contour $), 3,4,5,6$, and $7 \sigma\left(1 \sigma=0.050 \mathrm{mJy}^{\text {beam }}{ }^{-1}\right)$.

(WR 79a and WR 95). Detections classified as probable need further confirmation.

Flux densities and radio positions were obtained by fitting a two-dimensional Gaussian with the AIPS-JMFIT tool. In most of the detections the uncertainty in the radio position is less than $1^{\prime \prime}$. Except for WR 79a and WR 95, the optical and radio positions agree within $2^{\prime \prime}$. The flux density uncertainties quoted in the table are equal to the rms noise $(1 \sigma)$ of the images. Flux density uncertainties were derived from the images by taking into account a relatively large region near the detected sources. For the 14 undetected sources, the upper limits in flux density correspond to $3 \sigma$. Typically, the $1 \sigma$ level is in the range $0.025-0.084 \mathrm{mJy}$ beam $^{-1}$. In the cases of WR $79 \mathrm{a}$ and WR 89 the $1 \sigma$ level is $0.13 \mathrm{mJy}^{-1}$ beam $^{-1}$. The large rms noise associated with the detection of WR 89 is due to the displacement of this star about 1.'9 from the field center. The typical uncertainty in the radio position is 0.5 .

Table 2 lists the observed $3.6 \mathrm{~cm}$ flux densities and radio coordinates of the definite and probable detections, along with the optical coordinates from the catalog of van der Hucht (2001). Nondetected sources are also indicated at the bottom of the table. The latter have typical uncertainties of about 1 " (Leitherer et al. 1997).

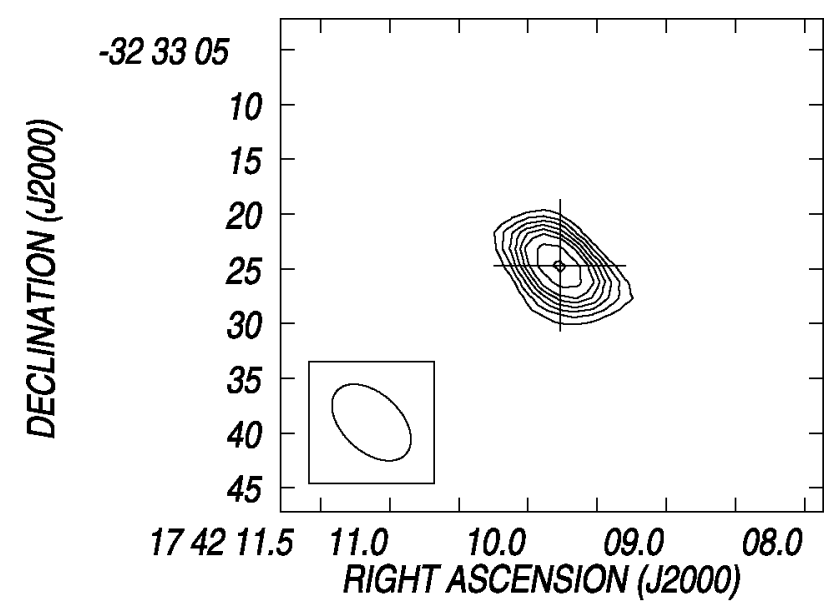

Fig. 10.-Contour image for WR 100. Contour lines are -3 (dashed contour $), 3,4,5,6,7,8,10$, and $12 \sigma\left(1 \sigma=0.040 \mathrm{mJy}\right.$ beam $\left.^{-1}\right)$.

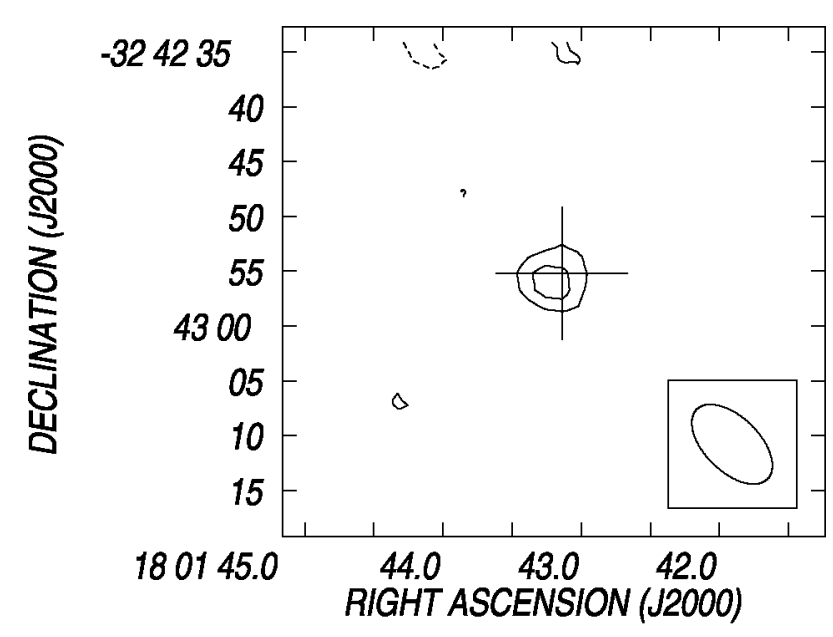

Fig. 11.-Contour image for WR 103. Contour lines are -3 (dashed contour $), 3$, and $4 \sigma\left(1 \sigma=0.040 \mathrm{mJy}^{\text {beam }}{ }^{-1}\right)$.

In Table 3 we compare our $3.6 \mathrm{~cm}$ detections with previously determined flux densities or upper limits at six radio wavelengths. The table also gives spectral indices $\alpha$ when calculable.

Since we performed observations at one frequency only, spectral indices cannot be derived from our data. Thus, the nature of the emission was inferred by comparing the observed fluxes with earlier determinations, allowing us to identify variable sources. Variability in flux density, which is a hint for the presence of nonthermal emission, is also indicated in Table 3 .

\section{COMMENTS ON INDIVIDUAL STARS}

WR 79a: WN9ha (Fig. 4). This object is a known visual binary with $\Delta \phi=6.9$ (Mason et al. 1998; van der Hucht 2001, Table 22). The radio source extends to the west. The earliest flux density determinations by BA89 (VLA) indicated thermal emission with a spectral index $\alpha=+0.8$. Recent ATCA observations ( 2000 November) by Setia Gunawan et al. (2003a) show a flat nonthermal energy distribution with spectral index $\alpha=-0.0 \pm 0.1$. Our $3.6 \mathrm{~cm}$ observation, less than a year later, is consistent with the ATCA observation and,

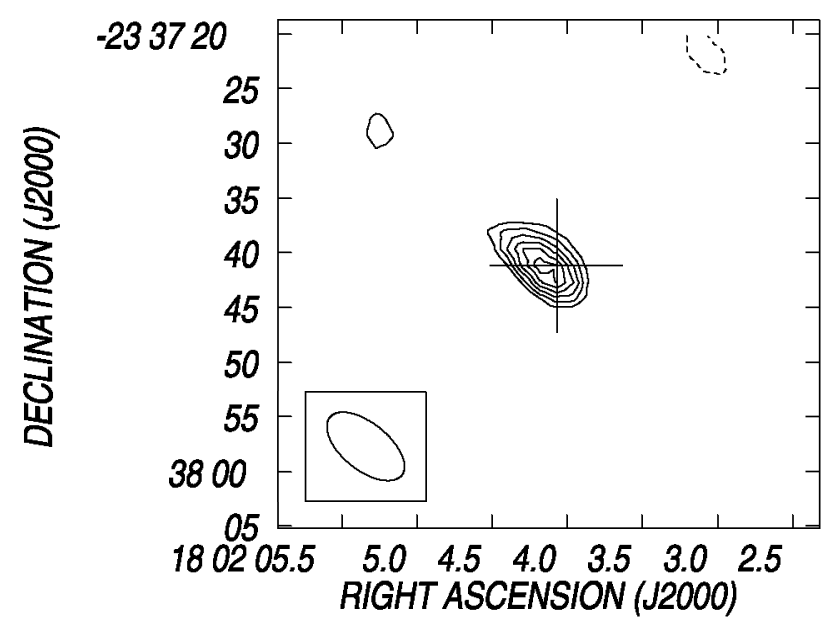

Fig. 12.-Contour image for WR 104. Contour lines are -3 (dashed contour $), 3,4,5,6,7$, and $8 \sigma\left(1 \sigma=0.060 \mathrm{mJy}\right.$ beam $\left.^{-1}\right)$. 


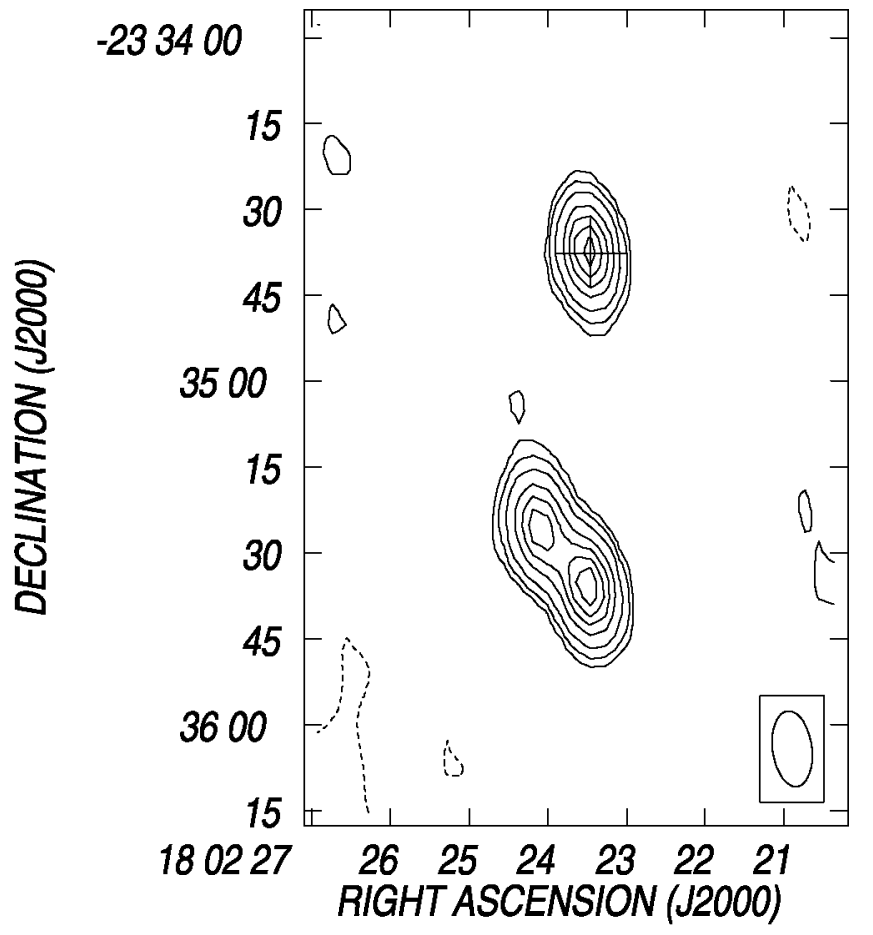

FIG. 13.-Contour image for WR 105. Contour lines are -3 (dashed contour), 3, 6, 12, 24, 36, 48, and $60 \sigma\left(1 \sigma=0.080 \mathrm{mJy} \mathrm{beam}^{-1}\right)$.

with respect to BA89 observation, with a variable nonthermal component.

WR 81: WC9 (Fig. 5). Our VLA image shows a source with a complex structure coincident with the optical position of the star. We identified the source to the north as the WR star because of its proximity ( 2 ".5) to the optical position. The radio source detected by AB 86 and identified by these authors as the WR star (see their Fig. 1) is 4"6 south of the optical position and closer to the southern extension of the radio source detected in the new image. A spectral index cannot be derived.

WR 89: WN8h+OB (Fig. 7). This source was detected in the image corresponding to WR $87,1.9$ displaced from the field center. Previous flux density determinations by LC95 were consistent with a thermal wind. Judging from our observation, the $3.6 \mathrm{~cm}$ flux density dropped some $30 \%$ in recent

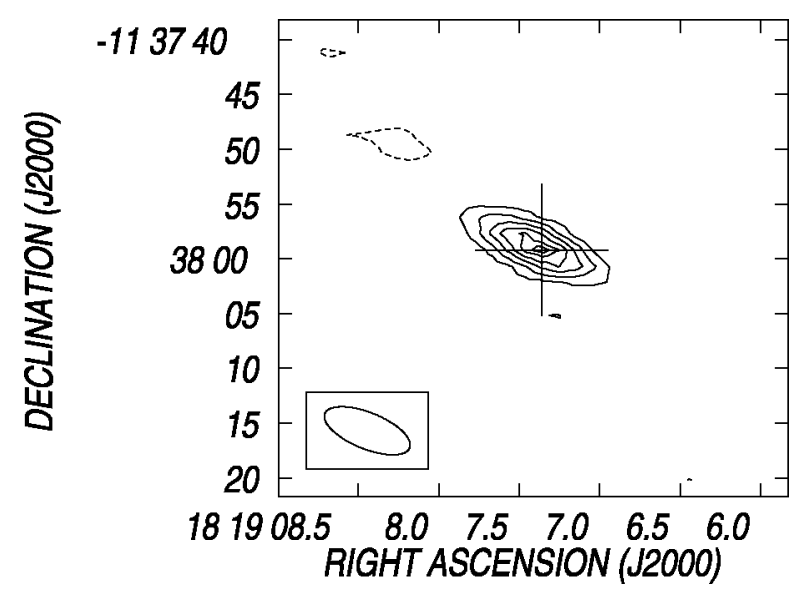

FIg. 14.-Contour image for WR 113. Contour lines are -3 (dashed contour $), 3,6,9,12,15$, and $17 \sigma\left(1 \sigma=0.040 \mathrm{mJy}^{-1}\right.$ beam $\left.^{-1}\right)$.

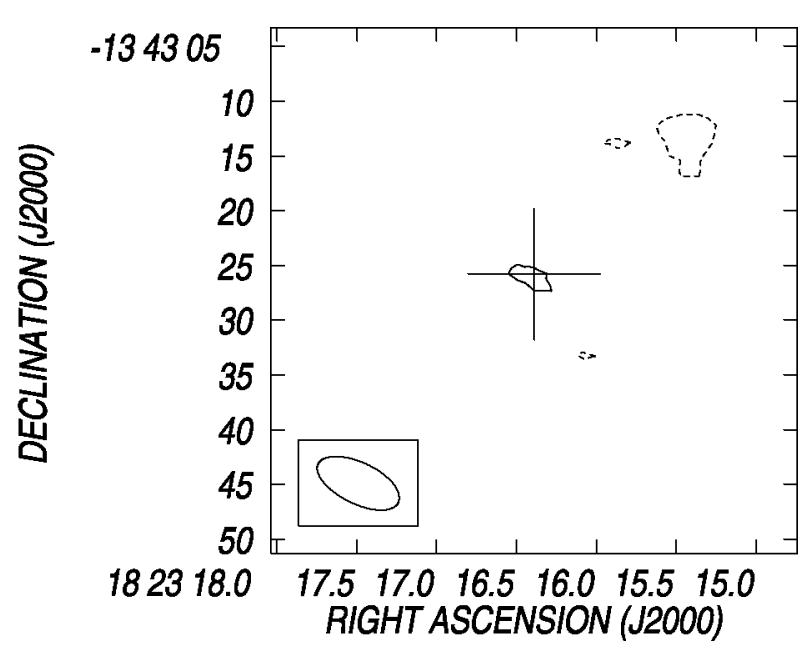

FIG. 15.-Contour image for WR 114. Contour lines are -3 (dashed contour $)$ and $3 \sigma\left(1 \sigma=0.040 \mathrm{mJy} \mathrm{beam}^{-1}\right)$.

years. WR 89 is associated with the open cluster HavlenMoffat No. 1 (C1715-387, Vázquez \& Baume 2001).

WR 98a: WC8-9vd+? (Fig. 9). In the infrared this object shows a expanding dust spiral, rotating with a period $P=$ $565 \pm 50$ days (Monnier et al. 1999), which is considered to be the binary period. Monnier et al. (2002) observed this object with the VLA in 1999.7 and 2000.2 and found the source to be nonthermal. Our $3.6 \mathrm{~cm}$ flux density determination is $\sim 20 \%$ below their result. Apparently, the nonthermal source is variable.

WR 104: WC9d+B0.5 V (Fig. 12). In the infrared this object shows a expanding dust spiral, rotating with a period $P=243 \pm 3$ days (Tuthill et al. 1999), which is considered to be the binary period. Monnier et al. (2002) found the source to be nonthermal. Our $3.6 \mathrm{~cm}$ flux density determination is $\sim 40 \%$ below theirs; thus, the nonthermal source is apparently variable.

WR 105: WN9h (Fig. 13). This star shows variable nonthermal flux densities. Our $3.6 \mathrm{~cm}$ flux density is $\sim 50 \%$ larger than the one measured by LC97. The double radio source to the south of the optical position of WR 105 was also detected by AB86 (see their Fig. 1). These radio sources were not detected in the NVSS (Condon et al. 1998), nor were optical

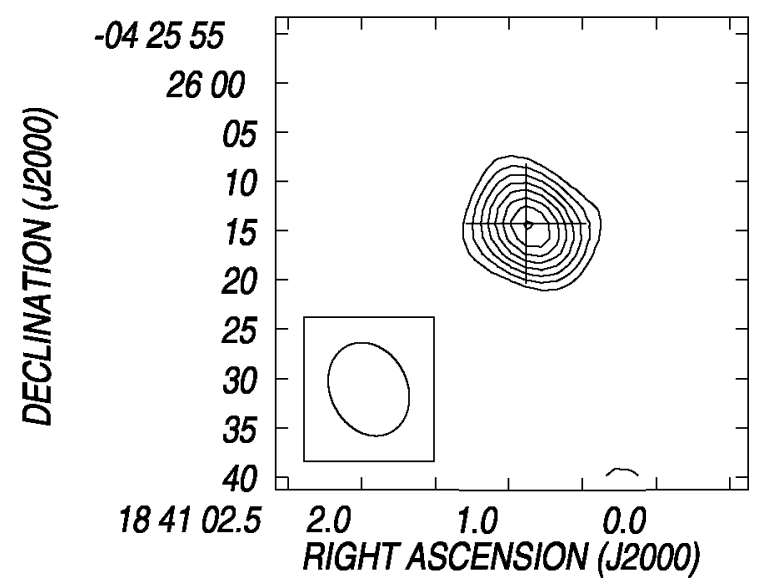

FIG. 16.-Contour image for WR 120. Contour lines are -3 (dashed contour $), 3,4,5,6,7,8,9$, and $10 \sigma\left(1 \sigma=0.035 \mathrm{mJy} \mathrm{beam}{ }^{-1}\right)$. 


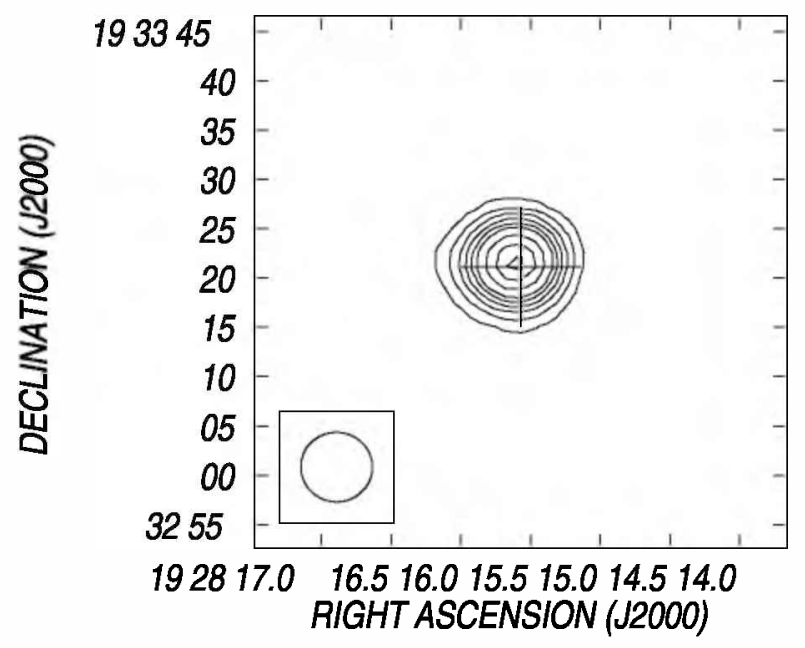

FIG. 17.-Contour image for WR 125. Contour lines are -3 (dashed contour $), 3,6,9,12,15,18,24,30$, and $35 \sigma\left(1 \sigma=0.030 \mathrm{mJy}^{\text {beam }}{ }^{-1}\right)$.

counterparts found. Zoonematkermani et al. (1990, radio continuum at $20 \mathrm{~cm}$ ) detected the double source with flux densities of $22 \mathrm{mJy}$ and $16 \mathrm{mJy}$, while from our VLA data we estimate $6.4 \pm 0.2$ and $5.4 \pm 0.2 \mathrm{mJy}$. These nonthermal sources have spectral indices $\alpha$ of -0.7 and -0.6 , respectively.
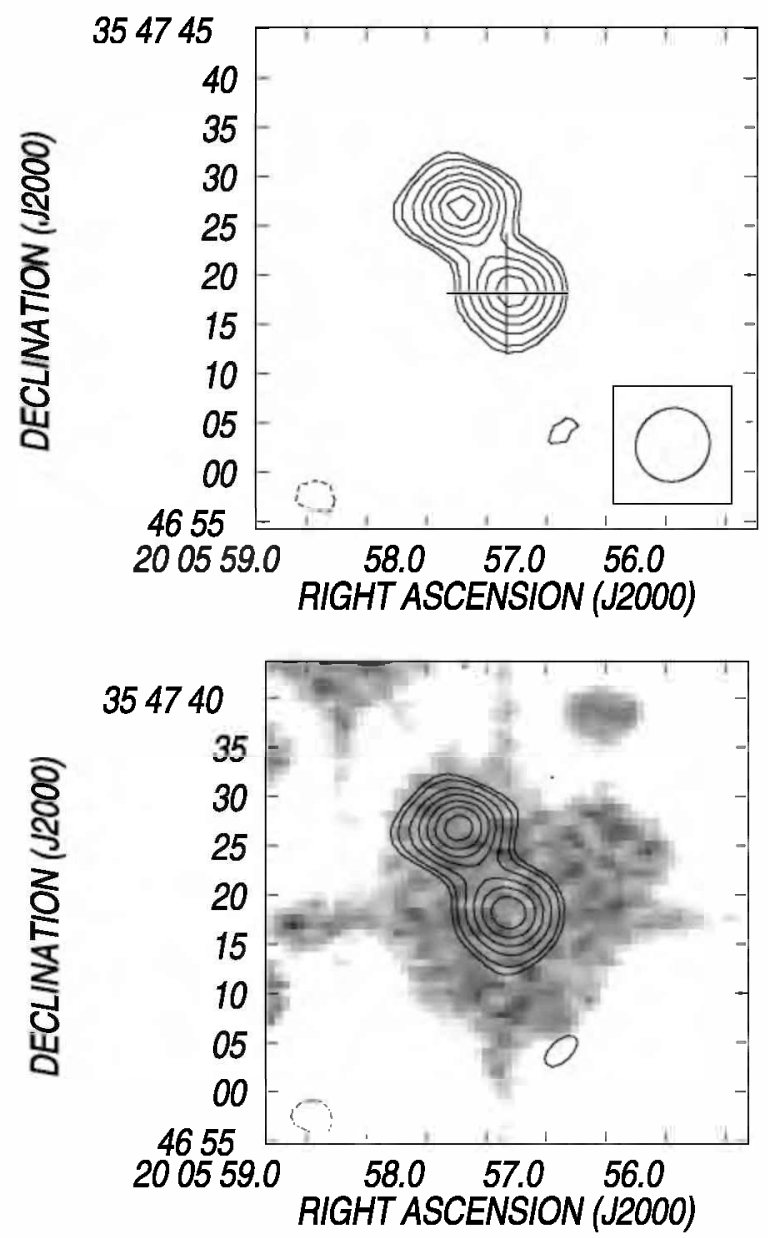

FIG. 18.-Contour image for WR 133. Top: Contour lines are -3 (dashed contour $), 3,4,6,8,10,12$, and $14 \sigma\left(1 \sigma=0.025 \mathrm{mJy}^{-1}\right.$ beam $\left.^{-1}\right)$. Bottom: Overlay of the DSS2 red image of WR 133 (gray scale, in arbitrary units) and the radio continuum emission at $3.6 \mathrm{~cm}$.

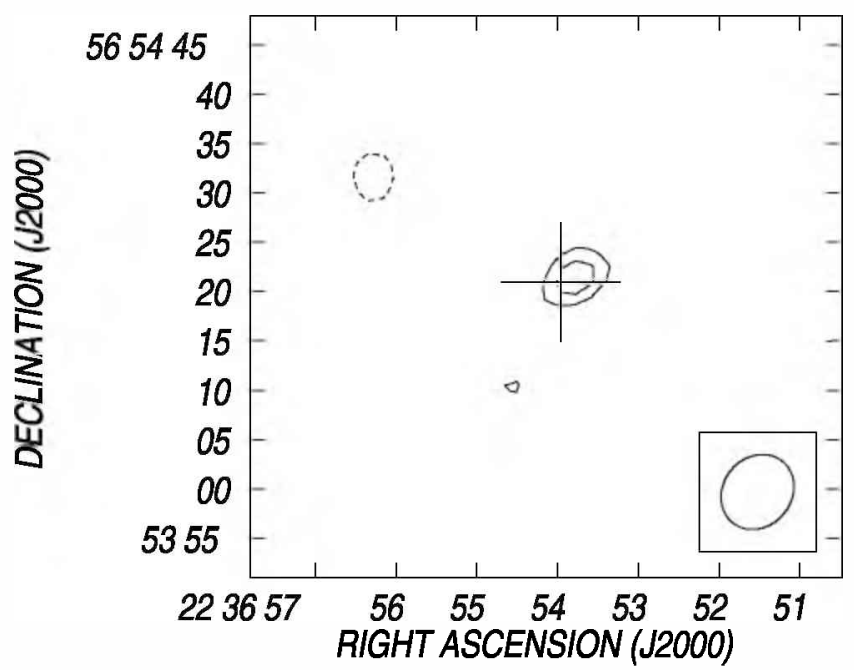

Fig. 19.-Contour image for WR 155. Contour lines are -3 (dashed contour $), 3$, and $4 \sigma\left(1 \sigma=0.025 \mathrm{mJy}^{\text {beam }}{ }^{-1}\right)$.

WR 125: WC7ed+O9 III (Fig. 17). The AB86 1982 observations at 6.3 and $2.0 \mathrm{~cm}$ showed a nonthermal radio source with $\alpha=-0.5$. Our $20013.6 \mathrm{~cm}$ flux density observation agrees with the AB86 data for a nonthermal source with that spectral index. The source is variable in flux and turned from nonthermal to thermal in WH92 (see Table 3). We suggest here that the emission is again nonthermal because of the similarity in flux density levels between the AB86 and the present data. The detection of nonthermal emission in 1982 and in 2001 suggests a period of around $20 \mathrm{yr}$. On the other hand, the last IR excess of the $\mathrm{WC}+\mathrm{O}$ binary WR 125 started in 1991 (WH92), continued till 1993, and then faded away. IR monitoring performed during the last $18 \mathrm{yr}$ showed only one period of infrared excess (Williams 2002), implying an orbital period of at least $18 \mathrm{yr}$. WR 125 has much in common with the archetype WR colliding wind binary WR 140 (HD 193793, WC7pd+O4-5, Williams et al. 1990), which has a thermal to nonthermal radio light curve with a period of $7.94 \mathrm{yr}$ (Williams et al. 1994). If WR 125 is an analog of WR 140, then its 1992 IR excess occurred at

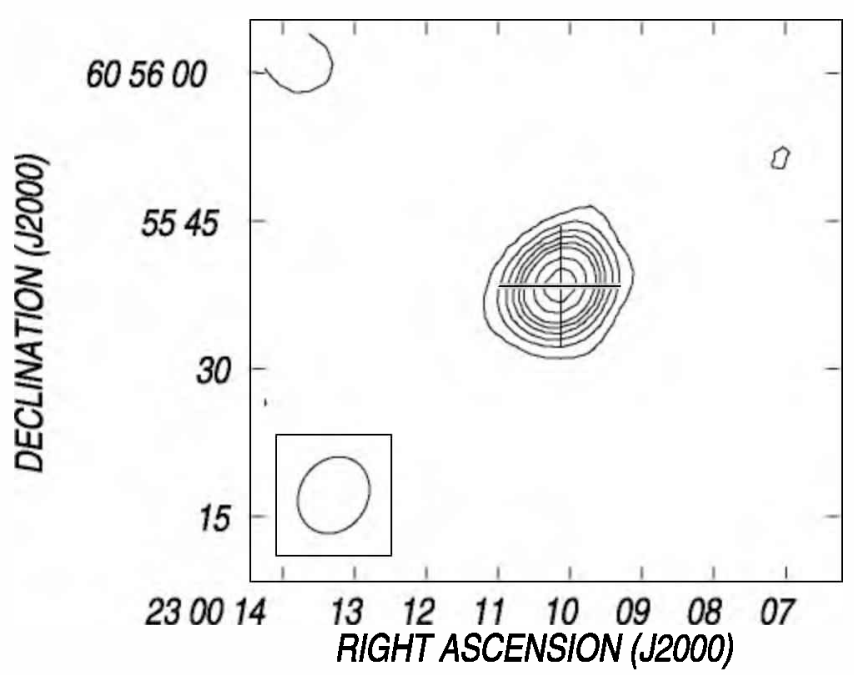

FIG. 20.-Contour image for WR 156. Contour lines are -3 (dashed contour $), 3,6,9,12,15,18,24$, and $30 \sigma\left(1 \sigma=0.030 \mathrm{mJy}^{\mathrm{J}}\right.$ beam $\left.^{-1}\right)$. 
TABLE 2

Optical Positions, Radio Positions, and Flux Densities of the Observed Wolf-Rayet Stars

\begin{tabular}{|c|c|c|c|c|c|c|}
\hline \multirow[b]{2}{*}{ WR No. } & \multicolumn{2}{|c|}{ Optical Position (J2000.0) } & \multicolumn{2}{|c|}{ Radio Position (J2000.0) } & \multirow{2}{*}{$\begin{array}{l}S_{3.6 \mathrm{~cm}} \\
(\mathrm{~m} J \mathrm{~J})\end{array}$} & \multirow[b]{2}{*}{ ЕРосH } \\
\hline & R.A. & Decl. & R.A. & Decl. & & \\
\hline \multicolumn{7}{|c|}{ Definite Detections } \\
\hline 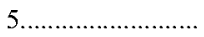 & 025211.66 & +565607.1 & $025211.62 \pm 0.05$ & $+565608.2 \pm 0.4$ & $0.20 \pm 0.03$ & 2001.9 \\
\hline $8 \ldots \ldots \ldots \ldots \ldots \ldots \ldots$ & 074458.22 & -315429.6 & $074458.26 \pm 0.02$ & $-315429.7 \pm 0.2$ & $0.46 \pm 0.03$ & 2001.8 \\
\hline $12 .$. & 084447.25 & -455855.5 & $084447.33 \pm 0.03$ & $-455852.5 \pm 0.7$ & $0.51 \pm 0.06$ & 2001.8 \\
\hline $88 \ldots \ldots \ldots \ldots$ & 171849.50 & -335739.8 & $171849.67 \pm 0.05$ & $-335740.9 \pm 0.6$ & $0.26 \pm 0.05$ & 2001.8 \\
\hline 89. & 171900.52 & -384851.2 & $171900.49 \pm 0.03$ & $-384849.4 \pm 0.4$ & $2.0 \pm 0.1$ & 2001.7 \\
\hline ................. & 174112.9 & -303229 & $174113.08 \pm 0.03$ & $-303230.0 \pm 0.3$ & $0.47 \pm 0.05$ & 2001.8 \\
\hline $100 \ldots \ldots \ldots \ldots \ldots \ldots$ & 174209.77 & -323324.7 & $174209.78 \pm 0.02$ & $-323324.9 \pm 0.2$ & $0.47 \pm 0.04$ & 2001.8 \\
\hline $103 \ldots \ldots \ldots \ldots \ldots \ldots$ & 180143.14 & -324255.2 & $180143.20 \pm 0.05$ & $-324255.7 \pm 0.7$ & $0.21 \pm 0.04$ & 2001.7 \\
\hline $104 \ldots \ldots \ldots \ldots \ldots \ldots \ldots$ & 180204.07 & -233741.2 & $180204.16 \pm 0.02$ & $-233741.0 \pm 0.3$ & $0.54 \pm 0.06$ & 2001.8 \\
\hline $105 \ldots \ldots \ldots \ldots \ldots \ldots$ & 180223.46 & -233437.7 & $180223.48 \pm 0.01$ & $-233437.3 \pm 0.3$ & $5.4 \pm 0.1$ & 2001.9 \\
\hline $113 \ldots \ldots \ldots \ldots \ldots \ldots$ & 181907.36 & -113759.2 & $181907.40 \pm 0.01$ & $-113758.9 \pm 0.1$ & $0.75 \pm 0.04$ & 2001.8 \\
\hline $120 \ldots \ldots \ldots \ldots \ldots \ldots$ & 184100.88 & -042614.3 & $184100.85 \pm 0.03$ & $-042614.6 \pm 0.4$ & $0.40 \pm 0.04$ & 2001.9 \\
\hline 125. & 192815.57 & +193321.1 & $192815.61 \pm 0.01$ & $+193321.6 \pm 0.1$ & $1.14 \pm 0.03$ & 2001.9 \\
\hline $133 \ldots \ldots \ldots \ldots \ldots \ldots$ & 200557.33 & +354718.2 & $200557.31 \pm 0.02$ & $+354718.6 \pm 0.3$ & $0.36 \pm 0.03$ & 2001.9 \\
\hline 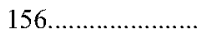 & 230010.13 & +605538.4 & $230010.14 \pm 0.01$ & $+605538.4 \pm 0.1$ & $1.06 \pm 0.03$ & 2001.9 \\
\hline \multicolumn{7}{|c|}{ Probable Detections } \\
\hline $79 \mathrm{a} \ldots \ldots \ldots \ldots \ldots$ & 165458.51 & -410903.1 & $165458.53 \pm 0.05$ & $-410907.8 \pm 0.8$ & $0.68 \pm 0.13$ & 2001.7 \\
\hline $81 \ldots \ldots \ldots \ldots \ldots \ldots \ldots$ & 170240.39 & -455915.5 & $170240.48 \pm 0.06$ & $-455912.9 \pm 1.4$ & $0.29 \pm 0.07$ & 2001.8 \\
\hline $95 \ldots \ldots \ldots \ldots \ldots \ldots$ & 173619.76 & -332610.9 & $173619.87 \pm 0.05$ & $-3326 \quad 13.4 \pm 0.7$ & $0.16 \pm 0.04$ & 2001.7 \\
\hline $114 \ldots \ldots \ldots \ldots \ldots \ldots$ & 182316.39 & -134325.8 & $182316.42 \pm 0.04$ & $-134325.9 \pm 0.2$ & $0.15 \pm 0.04$ & 2001.8 \\
\hline $155 \ldots \ldots \ldots \ldots \ldots \ldots$ & 223653.96 & +565421.0 & $223653.79 \pm 0.10$ & $+565421.5 \pm 0.7$ & $0.12 \pm 0.03$ & 2001.9 \\
\hline \multicolumn{7}{|c|}{ Undetected Sources } \\
\hline $4 \ldots \ldots$ & 024111.68 & +564349.7 & $\ldots$ & $\ldots$ & $<0.15$ & 2001.9 \\
\hline $79 \mathrm{~b} \ldots \ldots \ldots \ldots \ldots \ldots \ldots$ & 165506.45 & -445921.4 & $\ldots$ & $\ldots$ & $<0.20$ & 2001.7 \\
\hline $80 \ldots \ldots \ldots \ldots \ldots \ldots \ldots$ & 165902.2 & -454306 & $\ldots$ & $\ldots$ & $<0.17$ & 2001.8 \\
\hline $82 \ldots \ldots \ldots \ldots$ & 170404.61 & -451215.0 & $\ldots$ & $\ldots$ & $<0.23$ & 2001.7 \\
\hline $87 \ldots \ldots \ldots \ldots \ldots \ldots$ & 171852.89 & -385004.5 & $\ldots$ & $\ldots$ & $<0.24$ & 2001.7 \\
\hline $92 \ldots \ldots \ldots \ldots \ldots \ldots$ & 172523.15 & -432931.9 & $\ldots$ & $\ldots$ & $<0.18$ & 2001.8 \\
\hline $96 \ldots \ldots \ldots \ldots \ldots \ldots$ & 173624.2 & -325429 & $\ldots$ & $\ldots$ & $<0.16$ & 2001.7 \\
\hline $106 \ldots \ldots \ldots \ldots \ldots \ldots$ & 180443.66 & -210930.7 & $\ldots$ & $\ldots$ & $<0.17$ & 2001.8 \\
\hline $119 \ldots \ldots \ldots \ldots \ldots \ldots$ & 183917.91 & -100531.1 & $\ldots$ & $\ldots$ & $<0.11$ & 2001.7 \\
\hline $121 \ldots \ldots \ldots \ldots \ldots$ & 184413.15 & -034757.8 & $\ldots$ & $\ldots$ & $<0.17$ & 2001.9 \\
\hline $124 \ldots \ldots \ldots \ldots \ldots$ & 191130.88 & +165138.2 & $\ldots$ & $\ldots$ & $<0.25$ & 2001.9 \\
\hline $142 \ldots \ldots \ldots \ldots \ldots \ldots \ldots \ldots$ & 202144.36 & +372230.3 & $\ldots$ & $\ldots$ & $<0.90$ & 2001.9 \\
\hline $143 \ldots \ldots \ldots \ldots \ldots$ & 202822.68 & +383718.9 & $\ldots$ & $\ldots$ & $<0.12$ & 2001.9 \\
\hline $153 \mathrm{ab} \ldots \ldots \ldots \ldots \ldots$ & 221845.61 & +560733.9 & $\ldots$ & $\ldots$ & $<0.14$ & 2001.9 \\
\hline
\end{tabular}

NotE.- Units of right ascension are hours, minutes, and seconds, and units of declination are degrees, arcminutes, and arcseconds.

periastron passage in an eccentric orbit, and an IR excess will happen again in $\sim 2010-2012$.

WR 133: WN5+O9 I (Fig. 18). We detect a double pointlike source (separation $\sim 10^{\prime \prime}$ ) with the southern component coincident with the optical position of the WN5+O9 I binary (Fig. 18, top), which is a known visual binary $(\Delta \phi=5.4$, Hartkopf et al. 1999; van der Hucht 2001, Table 22). The source to the northeast has a flux density of $S_{3.6 \mathrm{~cm}}=0.39 \pm$ $0.03 \mathrm{mJy}$ beam $^{-1}$. The bottom panel of Figure 18 displays an overlay of the radio continuum emission and the DSS2 red image, suggesting the presence of an additional star northeast of the WR system, coincident in position with the northern radio source. Pollock et al. (1995) found a ROSAT X-ray excess for WR 133, indicative for a colliding wind binary.

Finally, we note that a radio source with a flux density $S_{3.6 \mathrm{~cm}}=0.15 \pm 0.04 \mathrm{mJy}$ is detected $2^{\prime \prime} .5$ distant from the optical position of WR 4 . We believe that this star has probably been barely detected, but, because of the low $\mathrm{S} / \mathrm{N}$ and the difference between the optical and radio positions $(2.1 \sigma)$, we have listed this star as nondetected.

In summary, we note that WR 79a, 89, 98a, 104, 105, and 125 are variable, which can be explained by the presence of a nonthermal component. This implies that our observations of these six stars cannot really be used for mass-loss rate determinations, or will yield only upper limits. Of the above six cases all three WCd objects (WR 98a, 104, and 125) turn out to be variable nonthermal radio sources, suggesting a large number of nonthermal cases between these objects. Additional observations are necessary to investigate the nature of the other three WCd stars in our sample (WR 95, 103, and 113) since previous observations only provided upper limits to the flux densities. 
TABLE 3

Radio Flux Densities of Earlier Detected Wolf-Rayet Stars, Compared with Our Detections

\begin{tabular}{|c|c|c|c|c|c|c|c|c|}
\hline \multirow[b]{2}{*}{ WR No. } & \multicolumn{6}{|c|}{ Present and Previous Observations (mJy) } & \multirow[b]{2}{*}{ Spectral IndeX $\alpha$} & \multirow[b]{2}{*}{ REFERENCE: } \\
\hline & $S_{21 \mathrm{~cm}}$ & $S_{12.5 \mathrm{~cm}}$ & $S_{6.3 \mathrm{~cm}}$ & $S_{3.6 \mathrm{~cm}}$ & $S_{2.0 \mathrm{~cm}}$ & $S_{1.3 \mathrm{~cm}}$ & & \\
\hline \multirow{3}{*}{ 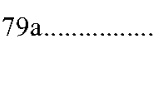 } & $\ldots$ & $\ldots$ & $1.1 \pm 0.1$ & & $2.4 \pm 0.1$ & $\ldots$ & +0.8 & BA89 \\
\hline & $\cdots$ & $1.0 \pm 0.1$ & $0.8 \pm 0.1$ & $0.9 \pm 0.1$ & $\ldots$ & $\cdots$ & -0.0 & $\mathrm{SC} 03$ \\
\hline & $\ldots$ & $\ldots$ & $\ldots$ & $0.7 \pm 0.1$ & $\ldots$ & $\ldots$ & $\mathrm{V}$ & This study \\
\hline \multirow[t]{2}{*}{ 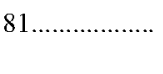 } & $\ldots$ & $\ldots$ & $0.3 \pm 0.08$ & $\ldots$ & $\ldots$ & $\ldots$ & $\ldots$ & AB86 \\
\hline & $\ldots$ & $\ldots$ & $\ldots$ & $0.3 \pm 0.1$ & $\ldots$ & $\ldots$ & $\ldots$ & This study \\
\hline \multirow{3}{*}{$88 \ldots \ldots \ldots \ldots \ldots . . . . . . . . . . .}$. & $\ldots$ & $\ldots$ & $<0.42$ & $<0.42$ & $\ldots$ & $\ldots$ & $\ldots$ & LC97 \\
\hline & $<1.29$ & $<0.57$ & $\ldots$ & $\ldots$ & $\ldots$ & $\ldots$ & $\ldots$ & CL99 \\
\hline & $\ldots$ & $\ldots$ & $\ldots$ & $0.26 \pm 0.05$ & $\ldots$ & $\ldots$ & $\ldots$ & This study \\
\hline \multirow[t]{4}{*}{$89 \ldots \ldots \ldots \ldots \ldots$} & $\ldots$ & $\ldots$ & $0.6 \pm 0.1$ & $\ldots$ & $\ldots$ & $\ldots$ & $\ldots$ & AB86 \\
\hline & $\ldots$ & $\ldots$ & $1.9 \pm 0.2$ & $3.0 \pm 0.1$ & $\ldots$ & $\ldots$ & +0.8 & LC95 \\
\hline & $<1.20$ & $<0.90$ & $\ldots$ & $\ldots$ & $\ldots$ & $\ldots$ & $\ldots$ & CL99 \\
\hline & $\ldots$ & $\ldots$ & $\ldots$ & $2.0 \pm 0.1$ & $\ldots$ & $\ldots$ & $\mathrm{V}$ & This study \\
\hline \multirow[t]{4}{*}{$95 \ldots$} & $\ldots$ & $\ldots$ & $<0.4$ & $\ldots$ & $\ldots$ & $\ldots$ & $\ldots$ & AB86 \\
\hline & $\cdots$ & $\cdots$ & $<0.45$ & $<0.45$ & $\ldots$ & $\cdots$ & $\cdots$ & LC97 \\
\hline & $<1.50$ & $<0.66$ & $\ldots$ & $\ldots$ & $\ldots$ & $\ldots$ & $\ldots$ & CL99 \\
\hline & $\ldots$ & $\ldots$ & $\ldots$ & $0.16 \pm 0.04$ & $\ldots$ & $\ldots$ & $\ldots$ & This study \\
\hline \multirow[t]{2}{*}{$98 \mathrm{a} . . . \ldots \ldots . . . . . . . .}$. & $<0.36$ & $\ldots$ & $0.37 \pm 0.07$ & $0.60 \pm 0.05$ & $0.64 \pm 0.11$ & $0.57 \pm 0.10$ & +0.3 & MT02 \\
\hline & $\ldots$ & $\ldots$ & $\ldots$ & $0.47 \pm 0.05$ & $\ldots$ & $\ldots$ & $\mathrm{V}$ & This study \\
\hline \multirow[t]{4}{*}{$103 \ldots \ldots \ldots \ldots$} & $\ldots$ & $\ldots$ & $\leq 0.2$ & $\ldots$ & $\ldots$ & $\ldots$ & $\ldots$ & AB86 \\
\hline & $\ldots$ & $\ldots$ & $<0.42$ & $<0.42$ & $\ldots$ & $\ldots$ & $\ldots$ & LC97 \\
\hline & $<0.90$ & $<0.42$ & $\ldots$ & $\ldots$ & $\ldots$ & $\ldots$ & $\ldots$ & CL99 \\
\hline & $\ldots$ & $\ldots$ & $\ldots$ & $0.21 \pm 0.04$ & $\ldots$ & $\ldots$ & $\ldots$ & This study \\
\hline \multirow[t]{5}{*}{$104 \ldots \ldots \ldots \ldots$} & $\ldots$ & $\ldots$ & $<0.4$ & $\ldots$ & $\ldots$ & $\ldots$ & $\ldots$ & $\mathrm{AB} 86$ \\
\hline & $\ldots$ & $\ldots$ & $<2.01$ & $<0.39$ & $\ldots$ & $\ldots$ & $\ldots$ & LC97 \\
\hline & $<1.59$ & $<0.99$ & $\ldots$ & $\ldots$ & $\ldots$ & $\cdots$ & $\cdots$ & CL99 \\
\hline & $<0.30$ & $\ldots$ & $\ldots$ & $0.87 \pm 0.06$ & $1.02 \pm 0.12$ & $0.94 \pm 0.10$ & +0.1 & MT02 \\
\hline & $\ldots$ & $\ldots$ & $\ldots$ & $0.54 \pm 0.06$ & $\ldots$ & $\ldots$ & $\mathrm{V}$ & This study \\
\hline \multirow[t]{4}{*}{$105 \ldots \ldots \ldots \ldots$} & $\ldots$ & $\ldots$ & $3.6 \pm 0.21$ & $\ldots$ & $\ldots$ & $\ldots$ & $\ldots$ & AB86 \\
\hline & $\ldots$ & $\ldots$ & $4.39 \pm 0.15$ & $3.8 \pm 0.2$ & $\ldots$ & $\ldots$ & -0.3 & LC97 \\
\hline & $<1.17$ & $<0.69$ & $\ldots$ & $\ldots$ & $\ldots$ & $\ldots$ & $\ldots$ & CL99 \\
\hline & $\ldots$ & $\ldots$ & $\ldots$ & $5.4 \pm 0.1$ & $\ldots$ & $\ldots$ & $\mathrm{V}$ & This study \\
\hline \multirow[t]{4}{*}{$113 \ldots \ldots \ldots \ldots \ldots$} & $\ldots$ & $\ldots$ & $\leq 0.4$ & $\ldots$ & $\ldots$ & $\ldots$ & $\ldots$ & BA82 \\
\hline & $\ldots$ & $\ldots$ & $<0.80$ & $<0.80$ & $\ldots$ & $\ldots$ & $\ldots$ & LC97 \\
\hline & $<2.25$ & $<0.90$ & $\ldots$ & $\ldots$ & $\ldots$ & $\ldots$ & $\ldots$ & CL99 \\
\hline & $\ldots$ & $\ldots$ & $\ldots$ & $0.75 \pm 0.04$ & $\ldots$ & $\ldots$ & $\ldots$ & This study \\
\hline \multirow{4}{*}{$114 \ldots \ldots \ldots \ldots \ldots$} & $\ldots$ & $\ldots$ & $<0.3$ & $\ldots$ & $\ldots$ & $\ldots$ & $\ldots$ & AB86 \\
\hline & $\cdots$ & $\cdots$ & $<0.45$ & $<0.45$ & $\cdots$ & $\cdots$ & $\cdots$ & LC97 \\
\hline & $<1.17$ & $<0.54$ & $\ldots$ & $\ldots$ & $\ldots$ & $\ldots$ & $\ldots$ & CL99 \\
\hline & $\ldots$ & $\ldots$ & $\ldots$ & $0.15 \pm 0.03$ & $\ldots$ & $\ldots$ & $\ldots$ & This study \\
\hline \multirow{4}{*}{$125 \ldots \ldots \ldots \ldots$} & $\ldots$ & $\ldots$ & $1.5 \pm 0.09$ & $\ldots$ & $0.8 \pm 0.09$ & $\ldots$ & -0.5 & AB86 \\
\hline & $1.53 \pm 0.06$ & $\cdots$ & $1.18 \pm 0.06$ & $\ldots$ & $0.82 \pm 0.10$ & $\cdots$ & -0.3 & WH92 \\
\hline & $\ldots$ & $\ldots$ & $0.20 \pm 0.04$ & $\ldots$ & $\ldots$ & $\ldots$ & $+0.7 \mathrm{~V}$ & WH92 \\
\hline & $\ldots$ & $\ldots$ & $\ldots$ & $1.14 \pm 0.03$ & $\ldots$ & $\ldots$ & $\ldots$ & This study \\
\hline \multirow[t]{2}{*}{$133 \ldots \ldots \ldots \ldots$} & $\ldots$ & $\ldots$ & $<0.3$ & $\ldots$ & $\ldots$ & $\ldots$ & $\ldots$ & AB86 \\
\hline & $\ldots$ & $\ldots$ & $\ldots$ & $0.36 \pm 0.03$ & $\ldots$ & $\ldots$ & $\ldots$ & This study \\
\hline
\end{tabular}

NoTE.-V: apparently variable.

References.-(AB86) Abbott et al. 1986; (BA82) Bieging, Abbott, \& Churchwell 1982; (BA89) Bieging et al. 1989; (CL99) Chapman et al. 1999; (LC95) Leitherer et al. 1995; (LC97) Leitherer et al. 1997; (MT02) Monnier et al. 2002; (SC03) Setia Gunawan et al. 2003a; (WH92) Williams et al. 1992.

\section{RADIO MASS-LOSS RATES}

Following Panagia \& Felli (1975) and Wright \& Barlow (1975), the flux density due to thermal Bremsstrahlung in an ionized expanding stellar wind can be expressed as

$$
S_{\nu}=2.32 \times 10^{4}\left(\frac{\dot{M} Z}{v_{\infty} \mu}\right)^{4 / 3}\left(\frac{\gamma g_{\mathrm{ff}} \nu}{d^{3}}\right)^{2 / 3},
$$

where $S_{\nu}$ is the flux density in millijanskys, $\dot{M}$ is the mass-loss rates in solar masses per year, $v_{\infty}$ is the terminal velocity in kilometers per second, $\nu$ is the frequency in hertz, and $d$ is the distance in kiloparsecs. The values $\mu, Z$, and $\gamma$ are the mean molecular weight, the rms ionic charge, and the mean number of electrons per ion, respectively. This expression corresponds to a spherically symmetric, stationary, isothermal wind flowing at constant velocity. The free-free Gaunt factor $g_{\mathrm{ff}}$ can be approximated, following Leitherer \& Robert (1991), as

$$
g_{\text {ff }}=9.77\left(1+0.13 \log \frac{T_{e}^{3 / 2}}{Z \nu}\right)
$$

In this expression $T_{e}$ is the electron temperature of the wind in kelvins. 
TABLE 4

Mass-Loss Rates from 3.6 Centimeter Observations of Our Sample of 34 Wolf-Rayet Stars

\begin{tabular}{|c|c|c|c|c|c|c|c|c|c|}
\hline WR No. & $\begin{array}{c}d \\
(\mathrm{kpc})\end{array}$ & $\begin{array}{c}v_{\infty} \\
\left(\mathrm{km} \mathrm{s}^{-1}\right)\end{array}$ & $\mu$ & $Z$ & $\gamma$ & $\begin{array}{l}S_{3.6 \mathrm{~cm}} \\
(\mathrm{~mJ})\end{array}$ & $\log \dot{M}$ & $\begin{array}{c}\dot{M} \\
\left(10^{-5} \mathrm{M}_{\odot} \mathrm{yr}^{-1}\right)\end{array}$ & Notes \\
\hline $4 \ldots \ldots \ldots \ldots \ldots$ & 2.44 & 1900 & 5.1 & 1.2 & 1.0 & $<0.15$ & $<-4.7$ & $<2.0$ & \\
\hline $5 \ldots \ldots \ldots \ldots \ldots$ & 1.91 & 2100 & 4.9 & 1.2 & 1.0 & $0.20 \pm 0.03$ & $-4.7 \pm 0.4$ & 1.8 & \\
\hline $8 \ldots \ldots \ldots \ldots \ldots$ & 3.47 & 1590 & 1.7 & 1.0 & 1.0 & $0.46 \pm 0.03$ & $-4.6 \pm 0.2$ & 2.6 & \\
\hline $12 \ldots \ldots \ldots \ldots$ & 5.04 & 1100 & 2.6 & 1.0 & 1.0 & $0.51 \pm 0.06$ & $-4.3 \pm 0.4$ & 5.2 & \\
\hline $79 \mathrm{a} \ldots \ldots \ldots \ldots$ & 1.99 & 935 & 2.6 & 1.0 & 1.0 & $0.68 \pm 0.14$ & $<-4.9 \pm 0.2$ & $<1.4$ & $\mathrm{NT} ?$ \\
\hline $79 \mathrm{~b} \ldots \ldots \ldots \ldots$ & 2.9 & 1650 & 2.6 & 1.0 & 1.0 & $<0.20$ & $<-4.8$ & $<1.7$ & \\
\hline $80 \ldots \ldots$ & 1.5 & 1200 & 4.7 & 1.1 & 1.1 & $<0.17$ & $<-5.2$ & $<0.6$ & \\
\hline $81 \ldots \ldots \ldots \ldots$ & 1.57 & 910 & 4.7 & 1.1 & 1.1 & $0.29 \pm 0.07$ & $-5.1 \pm 0.4$ & 0.8 & \\
\hline $82 \ldots \ldots \ldots \ldots$ & 5.25 & 1100 & 1.7 & 1.0 & 1.0 & $<0.23$ & $<-4.7$ & $<1.9$ & \\
\hline 87. & 2.88 & 1400 & 1.8 & 1.0 & 1.0 & $<0.24$ & $<-4.9$ & $<1.2$ & \\
\hline $88 \ldots \ldots \ldots \ldots$ & 2.33 & 1125 & 4.7 & 1.1 & 1.1 & $0.26 \pm 0.05$ & $-4.8 \pm 0.4$ & 1.6 & \\
\hline $89 \ldots \ldots \ldots \ldots$ & 2.88 & 1600 & 1.5 & 1.0 & 1.0 & $2.0 \pm 0.1$ & $-4.3 \pm 0.2$ & 5.2 & NT? \\
\hline $92 \ldots \ldots \ldots \ldots$ & 3.84 & 1100 & 4.7 & 1.1 & 1.1 & $<0.18$ & $<-4.6$ & $<2.5$ & \\
\hline $95 \ldots \ldots \ldots \ldots$ & 2.09 & 1100 & 4.7 & 1.1 & 1.1 & $0.16 \pm 0.04$ & $-5.0 \pm 0.2$ & 1.0 & \\
\hline $96 \ldots \ldots \ldots \ldots$ & 3.58 & 1100 & 4.7 & 1.1 & 1.1 & $<0.16$ & $<-4.7$ & $<2.1$ & \\
\hline $98 \mathrm{a} \ldots \ldots \ldots \ldots$ & 1.9 & 2000 & 4.7 & 1.1 & 1.1 & $0.47 \pm 0.05$ & $<-4.8 \pm 0.2$ & $<1.5$ & NT \\
\hline $100 \ldots \ldots \ldots \ldots$ & 4.61 & 1600 & 4.0 & 1.0 & 1.0 & $0.47 \pm 0.04$ & $-4.0 \pm 0.4$ & 9.5 & \\
\hline $103 \ldots \ldots \ldots \ldots$ & 2.21 & 1100 & 4.7 & 1.1 & 1.1 & $0.21 \pm 0.04$ & $-4.9 \pm 0.4$ & 1.2 & \\
\hline $104 \ldots \ldots \ldots \ldots$ & 2.3 & 1220 & 4.7 & 1.1 & 1.1 & $0.54 \pm 0.06$ & $<-4.5 \pm 0.2$ & $<2.9$ & NT \\
\hline $105 \ldots \ldots \ldots \ldots$ & 1.58 & 700 & 2.6 & 1.0 & 1.0 & $5.4 \pm 0.1$ & $<-4.5 \pm 0.4$ & $<3.4$ & NT \\
\hline $106 \ldots \ldots \ldots \ldots$ & 2.32 & 1100 & 4.7 & 1.1 & 1.1 & $<0.17$ & $<-5.0$ & $<1.1$ & \\
\hline $113 \ldots \ldots \ldots \ldots$ & 1.79 & 1700 & 4.7 & 1.1 & 1.1 & $0.75 \pm 0.04$ & $-4.4 \pm 0.2$ & 3.6 & \\
\hline $114 \ldots \ldots \ldots \ldots$ & 2.0 & 2000 & 4.9 & 1.2 & 1.1 & $0.15 \pm 0.04$ & $-4.8 \pm 0.2$ & 1.5 & \\
\hline $119 \ldots \ldots \ldots \ldots$ & 3.31 & 1200 & 4.7 & 1.1 & 1.1 & $<0.11$ & $<-4.8$ & $<1.5$ & \\
\hline $120 \ldots \ldots \ldots \ldots$ & 3.56 & 1225 & 4.0 & 1.0 & 1.0 & $0.40 \pm 0.04$ & $-4.4 \pm 0.4$ & 4.4 & \\
\hline $121 \ldots \ldots \ldots \ldots$ & 1.83 & 1100 & 4.7 & 1.1 & 1.1 & $<0.17$ & $<-5.1$ & $<0.8$ & \\
\hline $124 \ldots \ldots \ldots \ldots$ & 3.36 & 710 & 3.7 & 1.0 & 1.0 & $<0.25$ & $<-4.9$ & $<1.3$ & \\
\hline $125 \ldots \ldots \ldots \ldots$ & 3.06 & 2900 & 4.7 & 1.2 & 1.1 & $1.14 \pm 0.03$ & $<-3.8 \pm 0.4$ & $<17.1$ & NT \\
\hline $133 \ldots \ldots \ldots \ldots$ & 2.14 & 1800 & 4.0 & 1.1 & 1.1 & $0.36 \pm 0.03$ & $-4.6 \pm 0.2$ & 2.8 & \\
\hline $142 \ldots \ldots \ldots \ldots$ & 0.95 & 5500 & 5.1 & 1.2 & 1.1 & $<0.77$ & $<-4.3$ & $<4.5$ & \\
\hline $143 \ldots \ldots \ldots \ldots$ & 1.07 & 2750 & 5.1 & 1.2 & 1.0 & $<0.12$ & $<-5.2$ & $<0.7$ & \\
\hline $153 \mathrm{ab} \ldots \ldots \ldots$ & 2.75 & 1785 & 4.0 & 1.0 & 1.0 & $<0.14$ & $<-4.7$ & $<<2.0$ & \\
\hline $155 \ldots \ldots \ldots \ldots$ & 2.75 & 1400 & 4.0 & 1.0 & 1.0 & $0.12 \pm 0.03$ & $-4.9 \pm 0.2$ & 1.4 & \\
\hline $156 \ldots \ldots \ldots \ldots$ & 3.56 & 660 & 3.3 & 1.0 & 1.0 & $1.06 \pm 0.03$ & $-4.4 \pm 0.2$ & 4.0 & \\
\hline
\end{tabular}

Based on equations (1) and (2), we have derived mass-loss rates $\dot{M}$ corresponding to the observed flux densities at $3.6 \mathrm{~cm}$, assuming that the observed radio emission is due to free-free emission. In the case of nonthermal contributions our values represent upper limits to the true mass-loss rate. All nondetections are indicated as upper limits to $\dot{M}$. Following LC97, we adopted $T_{e}=10^{4} \mathrm{~K}$ in expression (2). As pointed out by these authors, this temperature has a very minor effect on the $g_{\text {ffr }}$ Values for the mean molecular weight, the rms ionic charge, and the mean number of electrons per ion were taken from LC97. The values adopted for the WO star in our sample, WR 142, are those for the WC4 subtype (Crowther et al. 1998).

Table 4 lists the derived mass-loss rates $\dot{M}$ and upper limits. Uncertainties in these values come from several sources. Errors in mass-loss rate determinations were estimated following equation (5) in LC97. We adopted similar criteria as LC97 to estimate the uncertainty in each parameter. For the error in the distances, we assumed $20 \%$ in the case of cluster/associations distances and $50 \%$ in the case of field stars. The logarithmic uncertainty is then \pm 0.09 and \pm 0.25 , respectively. Errors in $g_{\text {ff }}$ are about $10 \%(0.05$ in logarithmic scale). We adopted uncertainties in wind terminal velocities of $10 \%$ (i.e., a logarithmic error of \pm 0.04 ). Finally, logarithmic errors in $Z, \gamma$, and $\mu$ were adopted as \pm 0.08 . Typical logarithmic errors in $\log \dot{M}$ are in the range \pm 0.20 to \pm 0.24 in case of cluster/association distances and \pm 0.40 in case of field stars. With the quoted uncertainties for the stellar distances and other wind parameters, uncertainties in massloss rates determinations are dominated by errors in distances, while uncertainties in flux densities have a minor impact.

Other sources of error are nonthermal contributions to the flux at $3.6 \mathrm{~cm}$. Inhomogeneities or clumping in the stellar winds lead to overestimates of the final values, as emphasized by, e.g., Contreras et al. (1996) and Morris et al. (2000). The latter found volume-filling factors in the range $0.04-0.25$, leading to mass-loss rates a factor of 2.5-5 lower than expected for smooth, homogeneous winds. These effects have not been considered here.

\section{DISCUSSION}

To compare mass-loss rates derived for different WR subtypes from available radio surveys, we plotted the ATCA and VLA mass-loss rates versus WR subtype in Figure 21. The ATCA surveys have been performed by LC95, LC97, and CL99. Previous VLA results were published by Willis (1991) (who updated AB86 results by taking into account new criteria in terminal velocities and in the chemical composition of the region where radio emission originates) and by Monnier et al. (2002). 

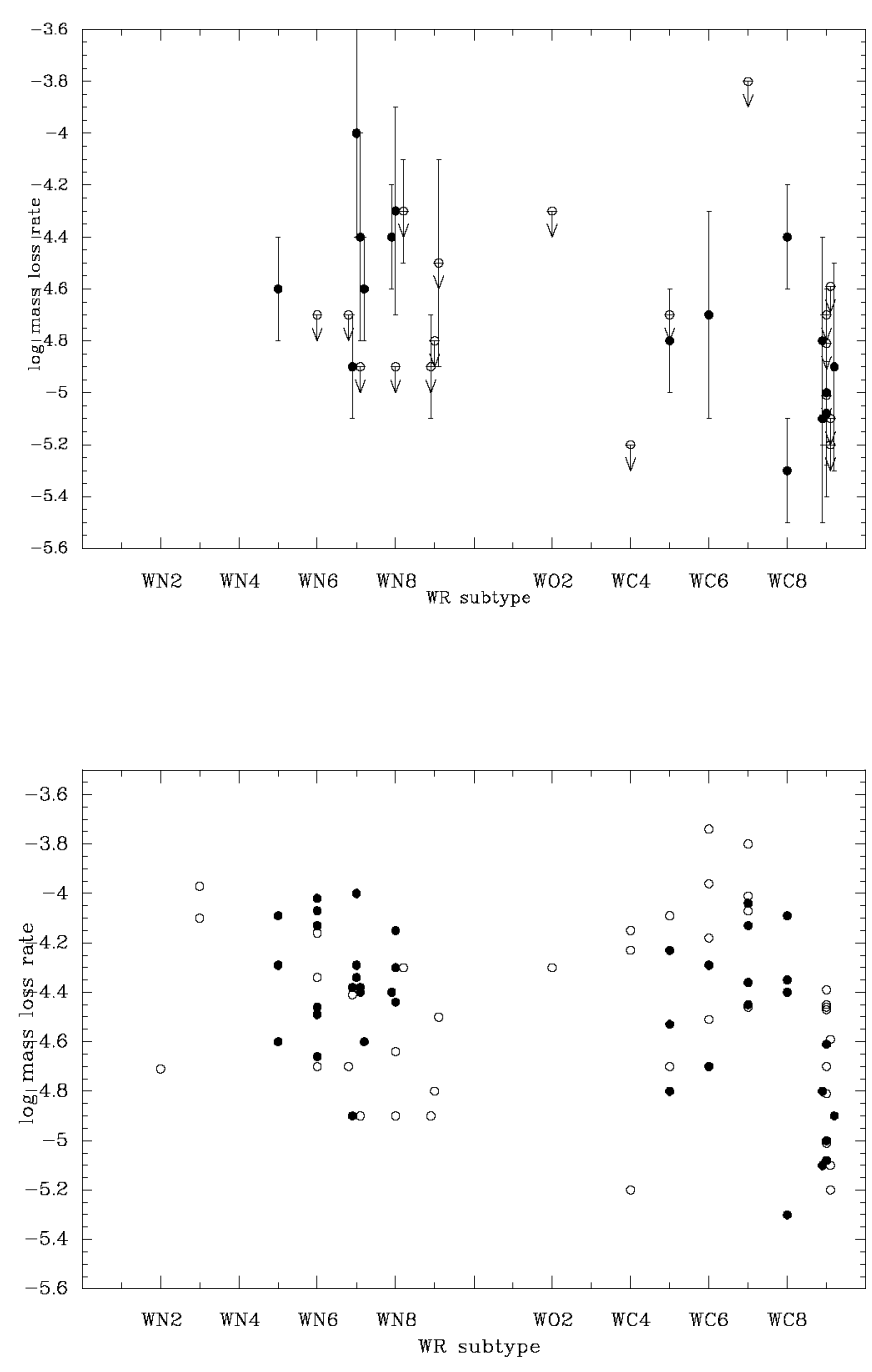

Fig. 21.-WR mass-loss rates $(\log \dot{M}$, where $\dot{M}$ is in units of solar masses per year) from radio observations vs. spectral subtype. Top: Mass-loss rates derived from the present VLA survey. Bona fide $M$-values corresponding to detected sources are indicated as filled circles. Bar errors are included. Open circles indicate upper limits corresponding to both detected sources with variable flux density and undetected sources. Bottom: Mass-loss rates derived for the VLA and ATCA target stars. Open and filled circles have the same meaning as in the top panel.

Monnier et al. observed WR 98a and WR 104 at several frequencies and found a nonthermal component in addition to the thermal one. They separated both components and derived a mass-loss rate from the best-fitting value to the thermal emission. The $\dot{M}$-values they derived are $0.8 \times 10^{-5} M_{\odot} \mathrm{yr}^{-1}$ for WR 98a and $0.5 \times 10^{-5} M_{\odot} \mathrm{yr}^{-1}$ for WR 104 .

The top panel of Figure 21 displays the mass-loss rate $(\log \dot{M})$ as a function of the spectral WR subtype for the stars included in our VLA survey. Bona fide mass-loss rate estimates are indicated by filled circles, while open circles indicate upper limits both for the variable detected sources and the undetected sources. Uncertainties in the derived values are also indicated. Note that we have plotted the mass-loss rates derived by Monnier et al. instead of our estimates listed in Table 4.

The bottom panel of Figure 21 shows all radio mass-loss rates from the ATCA and VLA samples. Although more than one mass-loss rate determination is available for some of the stars, we plotted only one value for each star from (in order of preference) Monnier et al. (2002), new VLA data, ATCA data, and previous VLA surveys. In the cases of nonthermal sources listed by LC97 and CL99, the $\dot{M}$-values plotted are upper limits. As in the top panel, open circles indicate upper limits both for undetected sources and variable detected sources.

Excluding definite and suspected nonthermal cases (WR $79 \mathrm{a}, 89$, and 105 ), the average mass-loss rate for the $\mathrm{WN}$ stars detected in our survey is $\dot{M}(\mathrm{WN})=(4.3 \pm 2.5) \times 10^{-5} M_{\odot}$ $\mathrm{yr}^{-1}$, equal to the average WN mass-loss rate in ATCA and previous VLA data sets of $\dot{M}(\mathrm{WN})=4 \times 10^{-5} M_{\odot} \mathrm{yr}^{-1}$.

Mass-loss rates determined from emission lines studies by Nugis \& Lamers (2000) differ from values derived from radio continuum data: in some cases (WR 104 and WR 113) $\dot{M}$ derived from emission lines are a factor of 2 smaller than our radio estimates, while in other cases (WR 81, 95, and 103) they are a factor of 2 larger than our results. Clumping and/or an ionization or temperature structure different from our assumptions may account for the differences.

We have 13 WC8-9 stars included in our sample and detected seven of them. Four of these stars had no radio detections yet (WR 88, 95, 103, and 113), thus increasing to 12 the number of WC8-9 stars with radio detections. Most of these stars have heated circumstellar dust shells (see Williams 1995). WR 95, 103 , and 113 are known to have persistent dust shells (see Table 1). The distribution of these stars in the diagram displays the same trend as in Figure 17 of LC97, with relatively low mass-loss rates. The average mass-loss rate for WC8-9 stars detected in our sample, excluding definite nonthermal cases (WR 98a and WR 104) is $\dot{M}$ (WC8-9) $=(1.7 \pm 1.0) \times 10^{-5} M_{\odot}$ $\mathrm{yr}^{-1}$. The average mass-loss rate for WC5-7 stars, obtained from bona fide values from the ATCA (five stars) and the previous (two stars) and present (one star) VLA samples, is $\dot{M}($ WC5 -7$)=(4.4 \pm 1.6) \times 10^{-5} M_{\odot} \mathrm{yr}^{-1}$, higher than the value derived for WC8 -9 stars. Note, on the other hand, that average values for WN and WC5-7 stars are equal. Uncertainties in stellar distances clearly contribute to the observed scatter of mass-loss rates.

The presence of dust in the wind envelopes might decrease the escaping UV photon flux, lowering the thermal radio emission, as suggested by Monnier et al. (2002), and thus the derived mass-loss rates. LC97 suggest that this may explain the low mass-loss rates obtained for WC9 stars from radio data. Note that the mass-loss rates for the WC8 star WR 98a, which also displays a dust persistent shell, is also relatively low (see Fig. 21, bottom).

The percentage of nonthermal emitters between the detected sources in our sample is $20 \%-30 \%$. A similar value is derived for the 1986 VLA survey (which also includes previous results). Our percentage is lower than the one derived from ATCA data $(40 \%)$.

The case of WR 89 (suggested to be a binary by van der Hucht 2001), which shows time variations in flux density (see Table 3), hints to additional nonthermal emission (the radio spectrum was thermal at the time of the ATCA observations). WR 79a also appears to be variable in flux, suggesting the presence of a nonthermal component. However, a variable thermal component cannot be discarded (e.g., Watson et al. 2002). It would be desirable to perform multifrequency radio observations of WR 79a and WR 89 to look for variability and to analyze the nature of the flux variations.

Finally, we would like to comment on the undetected stars. We repeat here that uncertainties in the expected flux densities $S_{\text {exp }}$ are quite large, arising mainly from uncertainties in distances of WR field stars and in the expected range of 
mass-loss rates $\left(1-5 \times 10^{-5} M_{\odot} \mathrm{yr}^{-1}\right) . S_{\exp }$ values for WR $79 \mathrm{~b}$, 92,96 , and 119 are $0.12-0.26,0.09-0.14,0.10-0.16$, and 0.11-0.16 mJy, respectively, which are below our detection limit (see Table 2). $S_{\text {exp }}$ values were estimated adopting the stellar parameters of Tables 1 and 4 , and mass-loss rates in the range $(1.5-2.0) \times 10^{-5} M_{\odot} \mathrm{yr}^{-1}$. For the cases of WR 80 and WR 121, however, $S_{\text {exp }}=0.5-0.8$ and $0.4-0.6 \mathrm{mJy}$, respectively. The derived upper limit is $0.17 \mathrm{mJy}(3 \sigma)$ for both stars. Although a mass-loss rate lower than typical cannot be discarded, the objects may be at larger distances than adopted here, thus lowering the expected flux density.

\section{CONCLUSIONS AND SUGGESTIONS FOR FUTURE WORK}

We performed a survey of 34 Galactic Wolf-Rayet stars at $3.6 \mathrm{~cm}$ within the declination range of the Very Large Array. We report 15 definite and five probable detections. Of these 20 sources, 13 were detected for the first time at radio frequencies.

We confirm time variations in flux of WR 98a, 104, 105, and 125, which support the presence of a nonthermal component. For WR 125 (WC7ed+O9 III) we suggest a binary period less than 20 yr. WR 79a (HD 152408, WN9ha) and WR 89 are also variable in flux, and we suspect they are also nonthermal emitters. Nonthermal radiation is indicative for wind-wind collision in $\mathrm{WR}+\mathrm{OB}$ binaries. Thus, of our sample $20 \%-30 \%$ of the detected stars are nonthermal radio sources.
Averages of mass loss rate determinations yield similar values for WN (all subtypes) and WC5-7 stars $[\dot{M}(\mathrm{WN})=$ $(4 \pm 3) \times 10^{-5} M_{\odot} \mathrm{yr}^{-1}$ and $\dot{M}(\mathrm{WC} 5-7)=(4 \pm 2) \times 10^{-5} M_{\odot}$ $\mathrm{yr}^{-1}$, while a slightly lower value was derived for WC8-9 stars $\left[\dot{M}(\mathrm{WC} 8-9)=(2 \pm 1) \times 10^{-5} M_{\odot} \mathrm{yr}^{-1}\right]$.

Future observations at several radio frequencies should monitor the objects WR 79a, 89, 98a, 104, and 125 during long time periods. These observations can help determine the presence of nonthermal contributions to the flux density as a function of time. Radio observations at low and high frequencies of the sources first detected will be useful to investigate the nature of the radio emission.

We thank the anonymous referee for very helpful comments and suggestions that improved the presentation of the paper. C. C. is grateful to the kind hospitality during her stay at AOC, NRAO in Socorro, New Mexico. The National Radio Astronomy Observatory is a facility of the National Science Foundation operated under cooperative agreement by Associated Universities, Inc. This research was partially supported by Facultad de Ciencias Astronómicas y Geofísicas, Universidad Nacional de La Plata; project 11/G049 (UNLP); and CONICET project PIP 607/98, Argentina. C. C. acknowledges a travel grant from Fundación Antorchas, Argentina, through project $13622 / 10$.

\section{REFERENCES}

Abbott, D. C., Bieging, J. H., Churchwell, E. B., \& Torres, A. V. 1986, ApJ, 303, 239 (AB86)

Bieging, J. H., Abbott, D. C., \& Churchwell, E. B. 1982, ApJ, 263, 207 1989, ApJ, 340, 518

Chapman, J. M., Leitherer, C., Koribalski, B., Bouter, R., \& Storey, M. 1999, ApJ, 518, 890 (CL99)

Churchwell, E. B., Bieging, J. H., van der Hucht, K. A., Williams, P. M., Spoelstra, T. A. Th., \& Abbott, D. C. 1992, ApJ, 393, 329

Condon, J. J., Cotton, W. D., Greisen, E. W., Yin, Q. F., Perley, R. A., Taylor, G. B., \& Broderick, J. J. 1998, AJ, 115, 1693

Contreras, M. E., Rodríguez, L. F., Gomez, Y., \& Velázquez, A. 1996, ApJ, 469,329

Crowther, P. A., De Marco, O., \& Barlow, M. J. 1998, MNRAS, 296, 367

Davis, R. J., Bode, M. F., Cohen, R. J., Dougherty, S. M., van der Hucht, K. A., \& Williams, P. M. 1996, in ASP Conf. Ser. 96, Radio Emission from the Stars and the Sun, ed. A. R. Taylor \& J. M. Paredes (San Francisco: ASP), 32

De Donder, E., \& Vanbeveren, D. 2003, NewA, 8, 415

Dougherty, S. M., Pittard, J. M., Kasian, L., Coker, R. F., Williams, P. M., \& Lloyd, H. M. 2003, A\&A, 409, 217

Dougherty, S. M., \& Williams, P. M. 2000, MNRAS, 319, 1005

Dougherty, S. M., Williams, P. M., \& Pollacco, D. L. 2000, MNRAS, 316, 143

Dougherty, S. M., Williams, P. M., van der Hucht, K. A., Bode, M. F., \& Davis, R. J. 1996, MNRAS, 280, 963

Dray, L. M., Tout, C. A., Karakas, A. I., \& Lattanzio, J. C. 2003, MNRAS, 338,973

Eenens, P. R. J., \& Williams, P. M. 1994, MNRAS, 269, 1082

Eichler, D., \& Usov, V. 1993, ApJ, 402, 271

Hartkopf, W. I., Mason, B. D., Gies, D. R., ten Brummelaar, T., McAlister, H. A., Moffat, A. F. J., Shara, M. M., \& Wallace, D. J. 1999, AJ, 118, 509

Kingsburgh, R. L., Barlow, M. J., \& Storey, P. J. 1995, A\&A, 295, 75

Lang, C. C. 2003, in IAU Symp. 212, A Massive Star Odyssey, From Main Sequence to Supernova, ed. K. A. van der Hucht, A. Herrero, \& C. Esteban (San Francisco: ASP), 497

Lang, C. C., Goss, W. M., \& Rodríguez, L. F. 2001, ApJ, 551, L143

Leitherer, C., Chapman, J. M., \& Koribalski, B. 1995, ApJ, 450, 289 (LC95) . 1997, ApJ, 481, 898 (LC97)

Leitherer, C., \& Robert, C. 1991, ApJ, 377, 629

Maeder, A. 1981, A\&A, 99, 97

Maeder, A., \& Conti, P. S. 1994, ARA\&A, 32, 227

Mason, B. D., Gies, D. R., Hartkopf, W. I., Bagnuolo, W. G., den Brummelaar, T., \& McAlister, H. A. 1998, AJ, 115, 821

Meynet, A., \& Maeder, A. 2003, A\&A, 404, 975
Monnier, J. D., Greenhill, L. J., Tuthill, P. G., \& Danchi, W. C. 2002, ApJ, 566, 399 Monnier, J. D., Tuthill, P. G., \& Danchi, W. C. 1999, ApJ, 525, L97

Moran, J. P., Davis, R. J., Bode, M. F., Taylor, A. R., Spencer, R. E., Argue, A. N., Irwin, M. J., \& Shanklin, J. D. 1989, Nature, 340, 449

Morris, P. M., van der Hucht, K. A., Crowther, P. A., Hillier, D. J., Dessart, L., Williams, P. M., \& Willis, A. J. 2000, A\&A, 353, 624

Nugis, T., \& Lamers, H. 2000, A\&A, 360, 227

Panagia, N., \& Felli, M. 1975, A\&A, 39, 1

Pollock, A. M. T., Haberl, F., \& Corcoran, M. F. 1995, in IAU Symp. 163, Wolf-Rayet Stars: Binaries, Colliding Winds, Evolution, ed. K. A. van der Hucht \& P. M. Williams (Dordrecht: Kluwer), 512

Prinja, R. K., Barlow, M. J., \& Howarth, I. D. 1990, ApJ, 361, 607 (erratum $383,466[1991])$

Setia Gunawan, D. Y. A., Chapman, J. M., Stevens, I. R., Rauw, G., \& Leitherer, C. 2003a, in IAU Symp. 212, A Massive Star Odyssey, From Main Sequence to Supernova, ed. K. A. van der Hucht, A. Herrero, \& C. Esteban (San Francisco: ASP), 230

Setia Gunawan, D. Y. A., de Bruyn, A. G., van der Hucht, K. A., \& Williams, P. M. 2000, A\&A, 356, 676

- 2001, A\&A, 368, 484 . 2003b, ApJS, 149, 123

Skinner, S. L., Itoh, M., Nagase, F., \& Zhekov, S. A. 1999, ApJ, 524, 394

Tuthill, P. G., Monnier, J. D., \& Danchi, W. C. 1999, Nature, 398, 487

van der Hucht, K. A. 2001, NewA Rev., 45, 135 2003, in IAU Symp. 212, A Massive Star Odyssey, From Main Sequence to Supernova, ed. K. A. van der Hucht, A. Herrero, \& C. Esteban (San Francisco: ASP), 441

van der Hucht, K. A., Williams, P. M., Spoelstra, T. A. Th., \& de Bruyn, A. G. 1992, in ASP Conf. Ser. 22, Nonisotropic and Variable Outflows from Stars, ed. L. Drissen, C. Leitherer, \& A. Nota (San Francisco: ASP), 253

Vázquez, R. A., \& Baume, G. 2001, A\&A, 371, 908

Watson, S. K., Davis, R. J., Williams, P. M., \& Bode, M. F. 2002, MNRAS, 334,631

White, R. L. 1985, ApJ, 289, 698

White, R. L., \& Becker, R. H. 1995, ApJ, 451, 352

Williams, P. M. 1995, in IAU Symp. 163, Wolf-Rayet Stars: Binaries, Colliding Winds, Evolution, ed. K. A. van der Hucht \& P. M. Williams (Dordrecht: Kluwer), 335

2002, in ASP Conf. Ser. 260, Interacting Winds from Massive Stars, ed. A. F. J. Moffat \& N. St-Louis (San Francisco: ASP), 311

Williams, P. M., Dougherty, S. M., Davis, R. J., van der Hucht, K. A., Bode, M. F., \& Setia Gunawan, D. Y. A. 1997, MNRAS, 289, 10 
Williams, P. M., van der Hucht, K. A., Bouchet, P., Spoelstra, T. A. Th., Eenens, P. R. J., Geballe, T. R., Kidger, M. R., \& Churchwell, E. B. 1992, MNRAS, 258, 461

Williams, P. M., van der Hucht, K. A., Pollock, A. M. T., Florkowski, D. R., van der Woerd, H., \& Wamsteker, W. M. 1990, MNRAS, 243, 662

Williams, P. M., van der Hucht, K. A., \& Spoelstra, T. A. Th. 1994, A\&A, 291,805

Williams, P. M., van der Hucht, K. A., van der Woerd, H., Wamsteker, W. M., Geballe, T. R., Garmany, C. D., \& Pollock, A. M. T. 1987, in Instabilities in Luminous Early Type Stars, ed. H. Lamers \& C. W. H. de Loore (Dordrecht: Reidel), 221
Willis, A. J. 1991, in IAU Symp. 163, Wolf-Rayet Stars: Binaries, Colliding Winds, Evolution, ed. K. A. van der Hucht \& P. M. Williams (Dordrecht: Kluwer), 265

Wright, A. E., \& Barlow, M. J. 1975, MNRAS, 170, 41

Zoonematkermani, S., Helfand, D. J., Becker, R. H., White, R. L., \& Perley, R. A. 1990, ApJS, 74, 181 